\title{
INTERIOR ESTIMATES FOR SECOND-ORDER DIFFERENCES OF SOLUTIONS OF FINITE-DIFFERENCE ELLIPTIC BELLMAN'S EQUATIONS
}

\author{
N. V. KRYLOV
}

\begin{abstract}
We establish interior estimates for the second-order finite differences of solutions of finite-difference approximations for uniformly elliptic Bellman's equations.
\end{abstract}

This article is a continuation of [15], where interior estimates for the first-order differences of solutions of finite-difference elliptic Bellman's equations are obtained. However, the present article can be read independently since we only use a few elementary facts from [15] and for that reason we repeat part of the introduction from [15]. We are dealing with the second-order differences of solutions of finitedifference equations of the type

$$
\sup _{\alpha \in A}\left[\sum_{|k|=1}^{d_{1}}\left[a_{k}^{\alpha}(x) \Delta_{h, l_{k}} v(x)+b_{k}^{\alpha}(x) \delta_{h, l_{k}} v(x)\right]-c^{\alpha}(x) v(x)+f^{\alpha}(x)\right]=0,
$$

where $x=\left(x^{1}, \ldots, x^{d}\right) \in \mathbb{R}^{d}, \Delta_{h, l_{k}} v$ and $\delta_{h, l_{k}} v$ are finite-difference approximations of the pure second-order derivative in the direction $l_{k}$ and the first-order derivative in the direction $l_{k}$, respectively (see (1.1)), $l_{k}, k= \pm 1, \ldots, \pm d_{1}$, are fixed vectors in $\mathbb{R}^{d}, h>0$ is the step size of finite differences, $d_{1} \geq d$ is a fixed integer, $A$ is a set, and $a_{k}^{\alpha}(x), b_{k}^{\alpha}(x), c^{\alpha}(x)$, and $f^{\alpha}(x)$ are given functions on $A \times \mathbb{R}^{d}$, the detailed assumptions on which will be given later.

For the reader more familiar with the theory of controlled Markov chains than with the theory of elliptic equations it is worth pointing out that under Assumption 1.1 the sum in (0.1) is rewritten as

$$
\sum_{l \in \Lambda} P_{h}^{\alpha}(x, l)[v(x+h l)-v(x)],
$$

where $\Lambda=\left\{l_{k}:|k|=1, \ldots, d_{1}\right\}$ and

$$
P_{h}^{\alpha}(x, l)=h^{-2} 2 a_{k}^{\alpha}(x)+h^{-1} b_{k}^{\alpha}(x)
$$

if $l=l_{k}$. In addition, $P_{h}^{\alpha}>0$ under Assumption 1.2.

Equation (0.1) is a finite-difference approximation for the following Bellman's equation arising, for instance, in the theory of controlled diffusion processes (see,

Received by the editor March 29, 2011 and, in revised form, November 25, 2011.

2010 Mathematics Subject Classification. Primary 35J60, 39A14.

Key words and phrases. Fully nonlinear elliptic equations, Bellman's equations, finite differences.

The author was partially supported by NSF Grant DMS-0653121. 
for instance, [4], 17]), convex geometry, and mathematical finance:

$$
\sup _{\alpha \in A}\left[L^{\alpha} v(x)-c^{\alpha}(x) v(x)+f^{\alpha}(x)\right]=0,
$$

where

$$
\begin{gathered}
L^{\alpha} v(x)=\sum_{i, j=1}^{d} a_{, i j}^{\alpha}(x) D_{i j} v(x)+\sum_{i=1}^{d} b_{, i}^{\alpha}(x) D_{i} v(x), \\
D_{i}=\frac{\partial}{\partial x^{j}}, \quad D_{i j}=D_{i} D_{j}, \\
a_{, i j}^{\alpha}(x)=\sum_{|k|=1}^{d_{1}} a_{k}^{\alpha}(x) l_{k}^{i} l_{k}^{j}, \quad b_{, i}^{\alpha}(x)=\sum_{|k|=1}^{d_{1}} b_{k}^{\alpha}(x) l_{k}^{i} .
\end{gathered}
$$

It is well known that for any uniformly elliptic operator of type (0.3) there exist constant vectors $l_{k}$ such that representation (0.4) holds. Here is Theorem 3.1 of [14]. Let $\mathcal{S}_{+}$be the set of $d \times d$ symmetric nonnegative matrices and for $\delta \in(0,1]$ let $\mathcal{S}_{\delta}$ be the subset of $\mathcal{S}$ consisting of matrices with eigenvalues in $\left[\delta, \delta^{-1}\right]$.

Theorem 0.1. There exists a set $\left\{\ell_{1}, \ldots, \ell_{n}\right\} \subset \mathbb{Z}^{d}$ such that for any its extension $\left\{\ell_{1}, \ldots, \ell_{d_{1}}\right\}, d_{1} \geq n$, there exist real-analytic functions $\lambda_{1}(a), \ldots, \lambda_{d_{1}}(a)$ on $\mathcal{S}_{\delta}$ possessing there the following properties:

$$
a \equiv \sum_{k=1}^{d_{1}} \lambda_{k}(a) \ell_{k} \ell_{k}^{*}, \quad \lambda_{k}(a) \geq \delta_{1}, \quad \forall k,
$$

where the constant $\delta_{1}>0$.

Hence, if we have an equation (0.2) with operators given in the form (0.3) and there is a $\delta>0$ such that $a^{\alpha}:=\left(a_{, i j}^{\alpha}(x)\right) \in \mathcal{S}_{\delta}$ for all $\alpha, x$, then the first equation in (0.4) holds with $a_{k}^{\alpha}(x)=(1 / 2) \lambda_{|k|}\left(a^{\alpha}\right)$ and $l_{k}=\ell_{|k|} \operatorname{sgn} k$. Notice that $a^{\alpha}(x)$ are as smooth as $a^{\alpha}(x)$. In this case, equation (0.2) is rewritten in terms of pure derivatives and constructing an approximation scheme like (0.1) becomes possible.

There are also many cases of degenerate fully nonlinear elliptic equations when such a reduction is possible (see, for instance, [13]). Of course, one of goals of passing from (0.2) to (0.1) is to give a method of numerical approximation of solutions of (0.2) in, say, bounded domains with Dirichlet boundary data. Concerning a quite long history of these methods we refer the reader to [1], 17] and the recent article 3.

It is proved in 3 and [11 that under some natural conditions the rate of convergence of numerical approximations to the true solution is $h^{1 / 2}$ as $h \downarrow 0$ and this rate is sharp if the equation may degenerate. This result is obtained on the basis of two main ideas from [8, 9, and [10] that the original equation and its finitedifference approximation should play symmetric roles and that one can "shake the coefficients" of the equation in order to be able to mollify under the sign of nonlinear operator. While shaking the coefficients of the approximate equation we encounter a major problem of estimating how much the solution of the shaken equation differs from the original one. Solving this problem amounts to estimating the Lipschitz constant of the approximate solution, which is achieved in [3] and 11 mainly by using a finite-difference version of Bernstein's method. 
The results in [3] and [1] are quite satisfactory in many situations, for instance, for degenerate elliptic equations, when one needs $c$ to be large enough, or for parabolic ones. However, if (0.2) is uniformly nondegenerate the requirement that $c$ be large enough looks very unnatural. The methods of [3] and [1] are not adapted to use the uniform nondegeneracy of the equation.

One of the goals of the present article is to give estimates of the second-order differences of approximate solutions without imposing any assumptions on the size of $c$. This allows us in [16] to improve the rate of convergence in 3] and 11] for uniformly nondegenerate equations.

What we mean is the following. Suppose that Assumptions 1.1, 1.2, and 1.3 stated in Section 1 are satisfied. Additionally assume that the coordinates of $l_{k}$ are rational numbers and $\operatorname{Span} \Lambda=\mathbb{R}^{d}$. Suppose that we are given a $\psi \in C^{2}\left(\mathbb{R}^{d}\right)$ such that

$$
\Omega:=\{x: \psi(x)>0\}
$$

is a bounded domain and $|D \psi| \geq 1$ on $\partial \Omega$. Introduce

$$
\Omega_{h}=\{x \in \Omega: x+h B \subset \Omega\}, \quad \partial_{h} \Omega=\mathbb{R}^{d} \backslash \Omega_{h},
$$

where $B$ is the unit ball centered at the origin.

The following is proved in 16 .

Theorem 0.2. (i) Equation (0.1) in $\Omega_{h}$ with zero boundary condition on $\partial_{h} \Omega$ has a unique bounded Borel solution $v_{h}$ if $h$ is small enough.

(ii) Equation (0.2) in $\Omega$ with zero boundary condition on $\partial \Omega$ has a unique solution $v \in C_{\text {loc }}^{1,1}(\Omega) \cap C^{0,1}(\bar{\Omega})$.

(iii) For all sufficiently small $h>0$ we have $\left|v_{h}-v\right| \leq N h^{2 / 3}$ on $\bar{\Omega}$, where $N$ is a constant.

To give an example of how our results can be applied to some concrete problems, take a strictly convex domain $\Omega$ with boundary of class $C^{4}$ and consider the equation

$$
\operatorname{det}\left(-D_{i j} v\right)=\left(f^{+}\right)^{d}
$$

in $\Omega$ with zero boundary condition, where $f^{+}$is the positive part of $f$. Solutions of (0.6) are sought in the class of concave functions, when the matrix $D^{2} v=\left(D_{i j} v\right)$ is negative definite. This is the so-called simplest Monge-Ampère equation. The reader can find how it is related to some problems in geometry, for instance, in [2.

It is well known (see, for instance, [5]) that equation (0.6) supplied with the requirement that $D^{2} v \leq 0$ is equivalent to the fact that $v$ satisfies

$$
\sup _{\alpha \in A}\left[\alpha_{i j} D_{i j} v+\left(\operatorname{det}^{1 / d} \alpha\right) f d\right]=0,
$$

where $A=\left\{\alpha \in \mathcal{S}_{+}: \operatorname{tr} \alpha=1\right\}$.

It is also known (see, for instance, [7]) that if $f \in C^{1,1}\left(\mathbb{R}^{d}\right)$, then (0.7) with zero boundary condition has a unique solution in $C^{1,1}(\bar{\Omega})$ and $\left|\operatorname{tr} D^{2} v\right| \leq N_{0}$, where the constant $N_{0}$ depends only on $\Omega$ and the $C^{1,1}$-norm of $f$. Equation (0.7) does not fit into our scheme because the matrices $\alpha \in A$ can be degenerate. However, if we are given that $f \geq 1$, then (0.6) and $\left|\operatorname{tr} D^{2} v\right| \leq N_{0}$ imply that all eigenvalues of $D^{2} v$ are less than $-N_{0}^{1-d}$. In that case one can disregard $\alpha \in A$ which are not in $\mathcal{S}_{\delta}$ where $\delta \in(0,1]$ is such that

$$
\delta^{1 / d} \sup f d<N_{0}^{1-d}
$$


because for those $\alpha$

$$
\alpha_{i j} D_{i j} v+\left(\operatorname{det}^{1 / d} \alpha\right) f d \leq-N_{0}^{1-d}+\delta^{1 / d} \sup f d<0 .
$$

It follows that $v$ also satisfies

$$
\sup _{\alpha \in A_{\delta}}\left[\alpha_{i j} D_{i j} v+\left(\operatorname{det}^{1 / d} \alpha\right) f d\right]=0,
$$

where $A_{\delta}=A \cap \mathcal{S}_{\delta}$, which owing to Theorem 0.1 fits into our scheme.

Notice that obtaining second-order difference estimates in a domain through the data and the maximum of the second-order differences on the boundary is useless from the point of view of improving the rates of convergence. Here the main obstacle is that, unlike the case of the first differences, there is apparently no way to estimate the second-order differences on the boundary. Therefore, the only useful information concerning the second-order differences is given by the interior estimates at least in what concerns the rate of convergence.

Our method is based on the ideas of Bernstein properly adapted to the case of finite-difference nonlinear equations. Bernstein's method exploits the maximum principle for a special function constructed from the second-order derivatives and the function itself and one of the main difficulties in the case of finite differences was to find such a combination which would be suitable for applying the maximum principle. We consider the maximum points of the expression

$$
\begin{gathered}
V_{\gamma \mu \nu}=\eta^{4}\left[\max \left(0,-\sum_{|k|=1}^{d_{1}} \gamma_{k} \Delta_{h, l_{k}} v\right)\right]^{2} \\
+\mu \eta^{4} \sum_{|k|,|j|=1}^{d_{1}}\left[\max \left(0,-\delta_{h, k} \delta_{h, j} v\right)\right]^{2}+\nu \eta^{2} \sum_{|k|=1}^{d_{1}}\left(\delta_{h, k} v\right)^{2},
\end{gathered}
$$

where $\gamma_{k}, \mu, \nu$ are some parameters and $\eta$ is a "cut-off" smooth auxiliary function. Note that the function $V_{\gamma \mu \nu}$ with $\eta \equiv 1$ was already introduced in [12] for the purposes of obtaining estimates from below for $\Delta_{h, l_{k}} v$.

In the implementation of the original Bernstein's method for Bellman's differential equations (see, for instance, [6]) one differentiates the equation twice in the direction of an arbitrary vector, say $\xi$, and obtains a differential inequality involving an elliptic operator for the second-order derivative $v_{(\xi)(\xi)}$ of $v$ in the direction of $\xi$. This inequality is then multiplied by $\max \left(0,-v_{(\xi)}(\xi)\right)$, which preserves the sign of the inequality and gives the possibility to obtain a further differential inequality for $\left[\max \left(0,-v_{(\xi)(\xi)}\right)\right]^{2}$. The latter and the maximum principle lead to an estimate of $\max \left(0,-v_{(\xi)(\xi)}\right)$ from above that is to an estimate of $v_{(\xi)(\xi)}$ from below. After that the estimate of $v_{(\xi)(\xi)}$ from above is obtained from the equation itself (cf. our Lemma 2.3).

Our first attempt to construct a combination of the second-order differences for which one could use the maximum principle was to consider

$$
\max \left(0,-\Delta_{h, l_{k}} v\right) .
$$

The main trouble with these functions was that after applying the operator $\Delta_{h, l_{k}}$ to (0.1) we get many terms containing mixed second and third finite-differences which we could not control. Then after a few other attempts we ended up with $V_{\gamma \mu \nu}$. It turns out that for $V_{\gamma \mu \nu}$ one can obtain meaningful inequalities at its maximum points provided they are inside the domain and an additional assumption (see (2.2)) 
holds. We say more about the underlying ideas in Remark 3.2 after we introduce appropriate notation.

The article is organized as follows. In Section 1 we present our main result, Theorem 1.1 and its discussion. We prove Theorem 1.1 in Section 2 relying on Theorem 2.1, which is proved in Section 3 with the help of Lemma 3.1, which in turn is proved in Section 5 preceded by Section 4 containing some technical tools.

Acknowledgment. The author is sincerely grateful to the referee for several useful comments.

\section{MAin RESUlt}

Let $A$ be a set and let

$$
a_{k}^{\alpha}=a_{k}^{\alpha}(x), \quad b_{k}^{\alpha}=b_{k}^{\alpha}(x), \quad c^{\alpha}=c^{\alpha}(x), \quad f^{\alpha}(x)
$$

be real-valued functions of $(\alpha, x)$ defined on $A \times \mathbb{R}^{d}$ for $k= \pm 1, \ldots, \pm d_{1}$. We assume that, for each $x$, these functions are bounded with respect to $\alpha \in A$. Also let some vectors $l_{k} \in \mathbb{R}^{d}$ be defined for $k= \pm 1, \ldots, \pm d_{1}$. For any $x, \xi \in \mathbb{R}^{d}, h>0$, and function $\phi$ on $\mathbb{R}^{d}$ introduce

(1.1) $T_{h, \xi} \phi(x)=\phi(x+h \xi), \quad \delta_{h, \xi}=h^{-1}\left(T_{h, \xi}-1\right), \quad \Delta_{h, \xi}=h^{-2}\left(T_{h, \xi}-2+T_{h,-\xi}\right)$.

When $\xi$ is one of $l_{k}$ 's we use the notation

$$
\delta_{h, k}=\delta_{h, l_{k}}, \quad \Delta_{h, k}=\Delta_{h, l_{k}}, \quad k= \pm 1, \ldots, \pm d_{1} .
$$

Assumption 1.1. We have $l_{k}=-l_{-k}, a_{k}^{\alpha}=a_{-k}^{\alpha}, k= \pm 1, \ldots, \pm d_{1}$, and

$$
0 \in \Lambda:=\left\{l_{k}, k= \pm 1, \ldots, \pm d_{1}\right\} .
$$

Observe that Assumption 1.1 and our other assumptions do not exclude the possibility that $l_{k}=0$ for some $k$. Our results still hold albeit the estimates of $\Delta_{h, k} v$ are trivial for those $k$ 's since $\Delta_{h, k} v=0$. Including the zero vector in $\Lambda$ turns out to be very convenient from a technical point of view. The coefficient $a_{k}^{\alpha}$ corresponding to this vector can be set to equal, say 1 , because the corresponding finite-difference operator is just zero. The assumption that $l_{k}=-l_{-k}, a_{k}^{\alpha}=a_{-k}^{\alpha}$ is also convenient from a technical point of view, since

$$
\Delta_{h, k}\left(\psi^{2}\right)=2 \psi \Delta_{h, k} \psi+\left(\delta_{h, k} \psi\right)^{2}+\left(\delta_{h,-k} \psi\right)^{2},
$$

whereas

$$
a_{k}^{\alpha} \Delta_{h, k}\left(\psi^{2}\right)=2 \psi a_{k}^{\alpha} \Delta_{h, k} \psi+2 a_{k}^{\alpha}\left(\delta_{h, k} \psi\right)^{2},
$$

where and everywhere below in the article the summation convention is enforced unless explicitly specified otherwise, which is very similar to what we have for second-order derivatives.

For any number $\gamma$ define

$$
\gamma^{ \pm}=(1 / 2)(|\gamma| \pm \gamma)
$$

Assumption 1.2. We are given three constants $\delta, K \in(0, \infty)$, and $h_{0} \in(0,1]$ such that for all $h \in\left(0, h_{0}\right], \alpha \in A$, and $k$ on $\mathbb{R}^{d}$ we have

$$
a_{k}^{\alpha} \geq \delta, \quad a_{k}^{\alpha} \geq 2 h_{0}\left(b_{k}^{\alpha}\right)^{-}, \quad c^{\alpha} \geq 0
$$

and for $\phi=a_{k}^{\alpha}, b_{k}^{\alpha}, c^{\alpha}, \alpha \in A$, and $i, j, k \in\left\{ \pm 1, \ldots, \pm d_{1}\right\}$ we have on $\mathbb{R}^{d}$ that

$$
|\phi| \leq K, \quad\left|\delta_{h, i} \phi\right| \leq K, \quad\left|\delta_{h, i} \delta_{h, j} \phi\right| \leq K
$$


Actually, we do not need (1.2) to be satisfied for all $h \in\left(0, h_{0}\right]$ since from now on we fix an $h \in\left(0, h_{0}\right]$ and we are going to use (1.2) only for this $h$. The above form of Assumption 1.2 was chosen to facilitate the statement of Theorem 0.2 .

In what follows we fix an $h \in\left(0, h_{0}\right]$ and set

$$
\Lambda_{1}=h \Lambda, \quad \Lambda_{n+1}=\Lambda_{n}+h \Lambda, \quad n \geq 1, \quad \Lambda_{\infty}=\bigcup_{n} \Lambda_{n},
$$

Fix a finite nonempty set $Q^{\circ} \subset \Lambda_{\infty}$, and define

$$
Q=\left\{x+\Lambda_{1}+\Lambda_{1}: x \in Q^{o}\right\}, \quad \partial Q=Q \backslash Q^{o} .
$$

Here, naturally, for instance,

$$
x+\Lambda_{1}+\Lambda_{1}=\left\{x+y+z: y, z \in \Lambda_{1}\right\}=\{x+h y+h z: y, z \in \Lambda\} .
$$

Next, for any function $\phi^{\alpha}(x)$ given on $A \times \mathbb{R}^{d}$ define

$$
\begin{gathered}
\left|\phi^{\prime}(x)\right|=\sup _{\alpha \in A, k}\left|\delta_{h, k} \phi^{\alpha}(x)\right|, \quad\left|\phi^{\prime \prime}(x)\right|=\sup _{\alpha \in A, j, k}\left|\delta_{h, j} \delta_{h, k} \phi^{\alpha}(x)\right|, \\
K_{0 \phi}=\sup _{x \in Q^{\circ}, \alpha \in A}\left|\phi^{\alpha}(x)\right|, \quad K_{1 \phi}=\sup _{Q^{\circ}}\left|\phi^{\prime}\right|, \quad K_{2 \phi}=\sup _{Q^{\circ}}\left|\phi^{\prime \prime}\right| .
\end{gathered}
$$

For functions $\phi(x)$ independent of $\alpha$ we also use the notation

$$
\left\|\phi^{\prime}\right\|=\sup _{\Lambda_{\infty}}\left|\phi^{\prime}\right|, \quad\left\|\phi^{\prime \prime}\right\|=\sup _{\Lambda_{\infty}}\left|\phi^{\prime \prime}\right| .
$$

In Theorem 1.1 and everywhere below we deal with fixed "cut-off" functions

$$
\eta \in C_{b}^{2}\left(\mathbb{R}^{d}\right), \quad|\eta| \leq 1, \quad \zeta=\eta^{2} .
$$

Before stating our main result about interior second-order differences estimates we introduce a structural assumption on the set of our basic vectors $\Lambda=\left\{l_{k}, k=\right.$ $\left.\pm 1, \ldots, \pm d_{1}\right\}$. For $d=2$ and the standard grid (generated by $\pm e_{1}, \pm e_{2}$ ) it means that $\Lambda$ contains all eight neighboring points of the origin on the grid.

Assumption 1.3. There exists an integer $1 \leq d_{0} \leq d_{1}$ such that for

$$
\mathcal{L}:=\left\{l_{ \pm 1}, \ldots, l_{ \pm d_{0}}\right\}
$$

we have $0 \in \mathcal{L}$ and

$$
\mathcal{L}+\mathcal{L} \supset \Lambda \supset\left\{l^{\prime}+l^{\prime \prime}: l^{\prime}, l^{\prime \prime} \in \mathcal{L}, l^{\prime} \neq l^{\prime \prime}\right\} .
$$

Remark 1.1. This is the correct version of Assumption 2.9 of [12, which is stated there somewhat ambiguously. Assumption 1.3 can be relaxed in the sense that the first inclusion can be replaced with $\mathcal{L}+\cdots+\mathcal{L} \supset \Lambda$, where on the left we have finitely many terms. This would only lead to the replacement of $\partial Q$ with a "thicker" set in our results. The only goal of this assumption is to allow one to estimate mixed partial differences $\delta_{h, i} \delta_{h, j} v$ through $\Delta_{h, k} v$ as in Lemma 2.2

Remark 1.2. We describe a way how to build a finite-difference scheme satisfying Assumptions 1.1, 1.2, and 1.3 for a given uniformly elliptic equation like (0.2) with operators $L^{\alpha}$ as in (0.3) (unrelated to (0.4) ).

Assume that there is a $\delta \in(0,1]$ such that $a^{\alpha}(x)=\left(a_{, i j}^{\alpha}(x)\right) \in \mathcal{S}_{\delta}$ for all $\alpha, x$ (uniform ellipticity) and that the functions $a^{\alpha}, b_{, i}^{\alpha}$, and $c^{\alpha}$ are of class $C^{1,1}\left(\mathbb{R}^{d}\right)$. Take the $\ell_{i}$ 's from Theorem 0.1 and let $\mathcal{L}$ be the set containing the zero vector, all 
$\pm \ell_{i}$, and all $\pm e_{j}$, where $e_{j}$ is the $j$ th basis vector in $\mathbb{R}^{d}$. Then set $\Lambda=\left\{l^{\prime}+l^{\prime \prime}\right.$ : $\left.l^{\prime}, l^{\prime \prime} \in \mathcal{L}, l^{\prime} \neq l^{\prime \prime}\right\}=:\left\{ \pm l_{1}, \ldots, \pm l_{d_{1}}\right\}$ and $l_{-k}=-l_{k}$. Theorem 0.1 implies that

$$
a^{\alpha}(x)=\sum_{|k|=1}^{d_{1}} a_{k}^{\alpha}(x) l_{k} l_{k}^{*}
$$

where $a_{k}^{\alpha}$ are in class $C^{1,1}\left(\mathbb{R}^{d}\right), a_{k}^{\alpha}=a_{-k}^{\alpha}$, and $a_{k}^{\alpha} \geq \delta_{1}$ with a constant $\delta_{1}>0$. After $a_{k}^{\alpha}$ have been defined introduce $b_{k}^{\alpha}=b_{i}^{\alpha}$ if $l_{k}=e_{i}$ and $b_{k}^{\alpha}=0$ if $l_{k} \notin\left\{e_{1}, \ldots, e_{d}\right\}$. For these defined objects our assumptions are obviously satisfied (with appropriate $\delta, K$, and $\left.h_{0}\right)$.

Remark 1.3. It is worth noting that the case $d_{0}=d_{1}$ can only happen if the set $\Lambda$ consists of only 0 or 0 and one more vector along with its negative. By the way, we do not assume that $\operatorname{Span} \Lambda=\mathbb{R}^{d}$.

A typical situation when one would apply Theorem 1.1 is when interior estimates for second-order differences are needed. Then one should choose $\eta$ vanishing in $\partial Q$, so that the first term on the right in (1.8) disappears.

Theorem 1.1. Suppose that the above assumptions are satisfied and a function $v$ given on $\mathbb{R}^{d}$ satisfies (0.1) in $Q^{\circ}$ and

$$
\sup _{\alpha \in A}\left[\sum_{|k|=1}^{d_{1}}\left[a_{k}^{\alpha}(x) \Delta_{h, l_{k}} v(x)+b_{k}^{\alpha}(x) \delta_{h, l_{k}} v(x)\right]-c^{\alpha}(x) v(x)+f^{\alpha}(x)\right] \leq 0
$$

on $\partial Q$. Then there exist constants $N$ and $N^{*}$ depending only on $K, d_{1}$, and $\delta$ such that if a constant $\nu>0$ satisfies

$$
\nu \geq N\left(\left\|\eta^{\prime \prime}\right\|+\left\|\eta^{\prime}\right\|^{2}\right)+N^{*}
$$

then

$$
\begin{aligned}
& \max _{Q, i, j} \zeta\left|\delta_{h, i} \delta_{h, j} v\right| \leq \max _{\partial Q, i, j} \zeta\left|\delta_{h, i} \delta_{h, j} v\right|+N\left(\nu^{-1} K_{2 f}+\nu^{-1 / 2} K_{1 f}+K_{0 f}\right) \\
& \quad+N\left[\nu^{1 / 2}+\left\|\eta^{\prime}\right\|+N^{*}\left(1+\nu^{-1}\right)\right] \max _{Q, i}\left|\delta_{h, i} v\right|+N^{*}\left(1+\nu^{-1}\right) \max _{Q}|v| .
\end{aligned}
$$

Furthermore, $N^{*}=0$ if $a_{k}^{\alpha}$ are independent of $x$ and $b=c=0$.

Remark 1.4. The author applies Theorem[1.1 in [16] as it is stated to investigate the rate of convergence of solutions of finite-difference schemes to the true solutions of Bellman's partial differential equations. In these situations it is possible to estimate

$$
\max _{Q, i}\left|\delta_{h, i} v\right|
$$

by means of Theorem 1.1 of $\left[15\right.$ (with $\eta \equiv 1$ there) and estimate $\delta_{h, i} v$ on the boundary by using simple barriers.

However, in some applications it is desirable to exclude (1.9) from the righthand side of (1.8). This can be done in two steps. If we are interested in estimating the second-order differences in a set $G^{o}$, where $G \subset Q$, take another subdomain $D \subset Q^{o}$ such that $G \subset D^{o}$ and take a cut-off function, which equals one in $G^{o}$ and vanishes outside $D^{o}$. This will allow one to estimate the second-order differences of the solution in $G^{o}$ through the first-order differences in $D$. The latter ones can be estimated through the maximum of the solution as noted before Theorem 1.1 of [15]. We give an example of this two-step procedure in the next remark. 
Remark 1.5. Theorem 1.1 allows one to obtain Liouville type theorems. Assume that $v$ satisfies (0.1) in $\Lambda_{\infty}, c=b=f=0, a$ is independent of $x$ and the sets $Q_{R}:=B_{R} \cap \Lambda_{\infty}$ are finite for any $R>0$, where $B_{R}=\{x:|x|<R\}$. By the way, the latter always holds if the coordinates of all $l_{k}$ are rational numbers. Also assume that

$$
\lim _{|x| \rightarrow \infty, x \in \Lambda} \frac{v(x)}{|x|^{2}}=0 .
$$

Then it turns out that $v$ is a linear function on $\Lambda_{\infty}$.

Indeed, take $\eta \in C_{0}^{\infty}\left(B_{1}\right)$ such that $\eta(0)=1$, take a large $R$, and apply Theorem 1.1 with $Q_{R}$ in place of $Q$ and $\eta_{R}(x):=\eta(x / R)$ in place of $\eta$. Then we obtain that

$$
\left|\delta_{h, i} \delta_{h, j} v(0)\right| \leq N R^{-1} \max _{Q_{R}, k}\left|\delta_{h, k} v\right|,
$$

On the other hand, take any $x_{0} \in Q_{R}$ and use Theorem 1.1 of [15] as in Remark 1.3 of [15]. Then we obtain that for large $R$,

$$
\left|\delta_{h, k} v\left(x_{0}\right)\right| \leq N R^{-1} \max _{x_{0}+Q_{R}}|v| \leq N R^{-1} \max _{Q_{2 R}}|v| .
$$

Hence,

$$
\left|\delta_{h, i} \delta_{h, j} v(0)\right| \leq N R^{-2} \max _{Q_{2 R}}|v| .
$$

Since the constants $N$ are independent of $R$, after letting $R \rightarrow \infty$ we get $\delta_{h, i} \delta_{h, j} v(0)$ $=0$. We can apply this argument to any point in $\Lambda_{\infty}$ and this shows that $v$ is linear on $\Lambda_{\infty}$ indeed.

This result is generally sharp since for the two-dimensional discrete Laplacian, say $L$, there are quadratic functions $v$ such that $L v=0$ on the standard grid.

To finish the section we introduce some abbreviated notation. The parameter $h$ is fixed throughout the paper. Also we will be dealing with a fixed solution $v$ of (0.1). Therefore, there is no ambiguity in setting

$$
\begin{gathered}
\delta_{k}=\delta_{h, k}=\delta_{h, l_{k}}, \quad \Delta_{k}=\Delta_{h, k}=\Delta_{h, l_{k}}, \\
v_{k}=\delta_{k} v, \quad v_{i k}=\delta_{i} \delta_{k} v .
\end{gathered}
$$

\section{Proof of Theorem 1.1}

We prove Theorem 1.1 under its assumptions on the basis of a few auxiliary results in the first of which the structural Assumption 1.3 is not needed. Also, for $\varepsilon \in(0,1]$ set

$$
\Gamma(\varepsilon)=\left\{\gamma=\left(\gamma_{k}: k= \pm 1, \ldots, \pm d_{1}\right): \gamma_{k}=\gamma_{-k}, \varepsilon \leq \gamma_{k} \leq \varepsilon^{-1}, \forall k\right\}
$$

and for $\gamma \in \Gamma(\varepsilon)$ set

$$
v_{\gamma}=\gamma_{k} \Delta_{k} v
$$

Everywhere below

$$
v_{\gamma}^{-}=\left(v_{\gamma}\right)^{-}, \quad v_{i j}^{-}=\left(v_{i j}\right)^{-} \quad\left(v_{i j}=\delta_{i} \delta_{j} v\right) .
$$

Here is a conditional version of Theorem 1.1. In Theorem 2.1 by $N$ we denote generic constants depending only on $\varepsilon, K, d_{1}$, and $\delta$ and by $N^{*}$ we denote those of $N$ 's which vanish if $a$ is independent of $x$ and $b \equiv c \equiv 0$. 
Theorem 2.1. Take $\gamma \in \Gamma(\varepsilon)$, fix a constant $\mu=\mu\left(d_{1}, \varepsilon\right)>0$ such that

$$
16 d_{1}^{2} \mu \leq \varepsilon^{2},
$$

and assume that

$$
3 \mu \max _{Q} \zeta^{2} \sum_{i, j}\left[v_{i j}^{-}\right]^{2} \leq \max _{Q} \zeta^{2}\left[v_{\gamma}^{-}\right]^{2} .
$$

Under these assumptions there exist constants $N \geq 1$ and $N^{*}$ such that, if a constant $\nu$ satisfies

$$
\nu \geq N\left(\left\|\eta^{\prime \prime}\right\|+\left\|\eta^{\prime}\right\|^{2}\right)+N^{*}
$$

then we have

$$
\begin{gathered}
\max _{Q, i, j} \zeta^{2}\left(v_{i j}\right)^{2} \leq \max _{\partial Q, i, j} \zeta^{2}\left(v_{i j}\right)^{2}+N\left(\nu^{-2} K_{2 f}^{2}+\nu^{-1} K_{1 f}^{2}\right) \\
+\left(N \nu+N^{*}\left(1+\nu^{-2}\right)\right) \bar{W}_{1}+N^{*}\left(1+\nu^{-2}\right) \bar{W}_{0}
\end{gathered}
$$

where

$$
W_{0}=v^{2}, \quad W_{1}=\sum_{i} v_{i}^{2}, \quad \bar{W}_{0}=\sup _{Q} W_{0}, \quad \bar{W}_{1}=\sup _{Q} W_{1} .
$$

We prove this theorem in Section 3

The following is a version of the elementary Lemma 7.1 of [12], which is proved there on the basis of Assumption 1.3

Lemma 2.2. For any $i, j \in\left\{ \pm 1, \ldots, \pm d_{1}\right\}$ and $x \in \Lambda_{\infty}$ we have

$$
\begin{gathered}
\left|\zeta(x) v_{i j}(x)\right| \leq N \max _{x+\Lambda_{2}}\left|\eta^{\prime}\right| \max _{x+\Lambda_{2}} W_{1}^{1 / 2} \\
+4 \max \left(\zeta(z)\left|\Delta_{k} v(z)\right|:|k| \leq d_{0}, z-x \in h \Lambda+h \mathcal{L}\right) \\
+4 \max \left(\zeta(z)\left|\Delta_{k} v(z)\right|:|k| \leq d_{1}, z-x \in h \Lambda\right),
\end{gathered}
$$

where $N$ is an absolute constant.

Proof. First we prove the lemma when $\zeta \equiv 1$. Observe that for any vectors $l^{\prime}, l^{\prime \prime}$ and function $\phi$,

$$
\begin{gathered}
\delta_{h, l^{\prime}} \delta_{h, l^{\prime \prime}} \phi(0)=(1 / 2)\left[\Delta_{h, l^{\prime \prime}} \phi\left(h l^{\prime}\right)+\Delta_{h, l^{\prime}} \phi\left(h l^{\prime \prime}\right)\right]-(1 / 2) \Delta_{h, l^{\prime}-l^{\prime \prime}} \phi(0), \\
\delta_{h, l^{\prime}} \delta_{h, l^{\prime}} \phi(0)=\Delta_{h, l^{\prime}} \phi\left(h l^{\prime}\right), \quad \delta_{h, l^{\prime}} \delta_{h,-l^{\prime}} \phi(0)=-\Delta_{h, l^{\prime}} \phi(0) .
\end{gathered}
$$
then

It follows from (2.6) and from the second inclusion in (1.5) that if $|i|,|j| \leq d_{0}$,

$$
\begin{aligned}
\left|v_{i j}(0)\right| & \leq \max \left(\left|\Delta_{k} v(z)\right|:|k| \leq d_{0}, z \in h \mathcal{L}\right) \\
& +\max \left(\left|\Delta_{k} v(0)\right|:|k| \leq d_{1}\right) .
\end{aligned}
$$

We substitute here $v(y+\cdot)$ in place of $v$. Then for $|i|,|j| \leq d_{0}$ we find that

$$
\begin{aligned}
\left|v_{i j}(y)\right| \leq & \max \left(\left|\Delta_{k} v(y+z)\right|:|k| \leq d_{0}, z \in h \mathcal{L}\right) \\
& +\max \left(\left|\Delta_{k} v(y)\right|:|k| \leq d_{1}\right),
\end{aligned}
$$

which for $y \in \Lambda_{1}$ implies that

$$
\begin{gathered}
\left|v_{i j}(y)\right| \leq \max \left(\left|\Delta_{k} v(z)\right|:|k| \leq d_{0}, z \in \Lambda_{2}\right) \\
+\max \left(\left|\Delta_{k} v(z)\right|:|k| \leq d_{1}, z \in \Lambda_{1}\right)=: I .
\end{gathered}
$$


In the general case by the first inclusion in (1.5) we have

$$
l_{i}=\xi_{1}+\xi_{2}, \quad l_{j}=\eta_{1}+\eta_{2}
$$

for some $\xi_{1}, \xi_{2}, \eta_{1}, \eta_{2} \in \mathcal{L}$. Introduce

$$
E:=\left\{\xi_{1}, \xi_{2}\right\} \cap\left\{\eta_{1}, \eta_{2}\right\}
$$

Observe that one of the following happens:

(i) $E$ is empty;

(ii) $E$ contains only one element;

(iii) $E$ consists of two points.

If $E$ is empty, then $\xi_{1} \neq \eta_{1}$ and in the formula

$$
\begin{gathered}
v_{i j}(0)=\delta_{h, l_{i}} \delta_{h, l_{j}} v(0)=\delta_{h, \xi_{2}} \delta_{h, \eta_{2}} v\left(h \xi_{1}+h \eta_{1}\right)+\delta_{h, \xi_{2}} \delta_{h, \eta_{1}} v\left(h \xi_{1}\right) \\
+\delta_{h, \xi_{1}} \delta_{h, \eta_{2}} v\left(h \eta_{1}\right)+\delta_{h, \xi_{1}} \delta_{h, \eta_{1}} v(0)
\end{gathered}
$$

we have $h \xi_{1}+h \eta_{1}, h \xi_{1}, h \eta_{1} \in \Lambda_{1}$, so that by the above $\left|v_{i j}(0)\right| \leq 4 I$. The same holds in case (ii) since in this case by relabeling $\xi_{i}, \eta_{j}$ if necessary we may assume that $\xi_{2}=\eta_{2}$ is the only point in $E$, so that again $\xi_{1} \neq \eta_{1}$. In case (iii) we have $l_{i}=l_{j}$ and (2.6) implies that

$$
\left|v_{i j}(0)\right| \leq \max \left(\left|\Delta_{k} v(z)\right|:|k| \leq d_{1}, z \in \Lambda_{1}\right) \leq I .
$$

This proves the lemma if $\zeta \equiv 1$.

To obtain (2.5) in the general case it suffices to observe that for $z \in x+\Lambda_{2}$ we have

$$
\left|(\zeta(x)-\zeta(z)) \Delta_{k} v(z)\right| \leq 2\left|(\eta(x)-\eta(z)) \Delta_{k} v(z)\right| \leq 4 \max _{x+\Lambda_{2}}\left|\eta^{\prime}\right| h\left|\Delta_{k}(z)\right|,
$$

where

$$
h\left|\Delta_{k}(z)\right|=\left|\delta_{k} v(z)+\delta_{-k} v(z)\right| \leq 2 \max _{x+\Lambda_{2}} W_{1}^{1 / 2} .
$$

The lemma is proved.

Taking Theorem 2.1 for granted for now, the main idea of the proof of Theorem 1.1 is to show that for an appropriate choice of $\varepsilon$ assuming that the opposite inequality in (2.2) holds for any $\gamma \in \Gamma(\varepsilon)$ allows one to estimate $\zeta v_{i j}$ from the equation itself. Lemma 2.2 shows that it suffices to estimate $\zeta \Delta_{k} v$.

Lemma 2.3. Let $\theta \in\left(0, \varepsilon^{-1}\right)$ and $\varepsilon \in(0,1]$ be such that

$$
K \kappa \leq \delta / 2, \quad \kappa:=\varepsilon(\theta+\varepsilon) /(1-\theta \varepsilon) .
$$

Assume that

$$
\max _{\Gamma(\varepsilon) \times Q} \zeta v_{\gamma}^{-} \leq \theta \max _{Q} \sum_{k} \zeta\left|\Delta_{k} v\right|
$$

Then in $Q$ we have

$$
\zeta \sum_{k}\left|\Delta_{k} v\right| \leq \frac{N\left(d_{1}\right)}{\delta}(1+\kappa)\left(K \bar{W}_{1}^{1 / 2}+K \bar{W}_{0}^{1 / 2}+K_{0 f}\right) .
$$

Proof. Set $\Phi^{( \pm)}=\zeta \sum_{k}\left(\Delta_{k} v\right)^{( \pm)}$and observe that, due to (2.8),

$$
\begin{gathered}
\varepsilon^{-1} \Phi^{(-)}-\varepsilon \Phi^{(+)} \leq \theta \max _{Q}\left(\Phi^{(+)}+\Phi^{(-)}\right), \\
\varepsilon \Phi^{(+)}-\varepsilon^{-1} \Phi^{(-)} \geq-\theta \max _{Q} \Phi^{(+)}-\theta \max _{Q} \Phi^{(-)}
\end{gathered}
$$


in $Q$. Hence $(\theta+\varepsilon) \max _{Q} \Phi^{(+)} \geq\left(\varepsilon^{-1}-\theta\right) \max _{Q} \Phi^{(-)}$, that is,

$$
\max _{Q} \Phi^{(-)} \leq \kappa \max _{Q} \Phi^{(+)} .
$$

Next, from equation (0.1) we find that in $Q$

$$
-K \Phi^{(-)}+\delta \Phi^{(+)}-N K \bar{W}_{1}^{1 / 2}-N K \bar{W}_{0}^{1 / 2}-K_{0 f} \leq 0,
$$

where and below in the proof the constants denoted by $N$ depend only on $d_{1}$. This, (2.10), and (2.7) imply that

$$
\max _{Q} \Phi^{(+)} \leq N \delta^{-1}\left(K \bar{W}_{1}^{1 / 2}+K \bar{W}_{0}^{1 / 2}+K_{0 f}\right),
$$

which after being combined with (2.10) yields (2.9) and proves the lemma.

It is worth noting a simple corollary of this lemma.

Corollary 2.4. If $b \equiv c \equiv f \equiv 0$, then the inequality opposite to (2.8) is true.

Indeed, if $b \equiv c \equiv f \equiv 0$ and (2.8) holds, the above proof shows that one can drop all terms on the right in (2.9). Then by (2.9) we have $\Delta_{k} v=0$ in $Q$ in which case the inequality opposite to (2.8) is true indeed.

Proof of Theorem 1.1. It is easy to see that one can find $\varepsilon=\varepsilon\left(\delta, d_{1}, K\right) \in(0,1]$ and $\mu=\mu\left(\delta, d_{1}, K\right) \in(0,1]$ in such a way that the conditions: (2.1),

$$
16 d_{1}^{2}(3 \mu)^{1 / 2}=: \theta<\varepsilon^{-1},
$$

and (2.7) are satisfied. We choose and fix appropriate $\varepsilon$ and $\mu$. We also take any $\nu$ from Theorem 2.1. Observe that $N \geq 1$ in (2.3). Hence $\left\|\eta^{\prime}\right\| \leq \nu^{1 / 2}$.

If condition (2.2) is satisfied, we obtain (1.8) by Theorem 2.1 If condition (2.8) is satisfied, we obtain (1.8) by Lemma 2.3 .

In the remaining case both (2.2) and (2.8) are violated, so that

$$
\begin{gathered}
\theta H:=\theta \max _{Q} \sum_{k} \zeta\left|\Delta_{k} v\right| \\
\leq \max _{\Gamma(\varepsilon) \times Q} \zeta v_{\gamma}^{-} \leq(3 \mu)^{1 / 2} \max _{Q} \zeta\left(\sum_{i, j}\left|v_{i j}\right|^{2}\right)^{1 / 2} \\
\leq(3 \mu)^{1 / 2} \max _{\partial Q} \sum_{i, j} \zeta\left|v_{i j}\right|+(3 \mu)^{1 / 2} \max _{Q^{o}} \sum_{i, j} \zeta\left|v_{i j}\right| .
\end{gathered}
$$

In light of Lemma 2.2 the last maximum over $Q^{\circ}$ is less than

$$
8 d_{1}^{2} H+N\left\|\eta^{\prime}\right\| \bar{W}_{1}^{1 / 2},
$$

where $N$ depends only on $d_{1}$. Since $(3 \mu)^{1 / 2} 8 d_{1}^{2}=\theta / 2$, we conclude

$$
\theta H \leq N \max _{\partial Q, i, j} \zeta\left|v_{i j}\right|+N\left\|\eta^{\prime}\right\| \bar{W}_{1}^{1 / 2} .
$$

Hence $H^{2}$ is dominated by the right-hand side of (1.8) and Lemma 2.2 implies that the same is true for the left-hand side of (1.8). The theorem is proved. 


\section{Proof of Theorem 2.1}

In this section the structural Assumption 1.3 is not used. Take any $\varepsilon, \mu \in(0,1]$, $\nu \in(0, \infty), \gamma \in \Gamma(\varepsilon)$, and introduce

$$
\begin{gathered}
P_{\gamma}=\gamma_{i} \Delta_{i}, \quad v_{\gamma}=P_{\gamma} v, \\
W_{1}=\sum_{i} v_{i}^{2}, \quad W_{2}=\sum_{i, j}\left[v_{i j}^{-}\right]^{2}, \quad V_{\gamma \mu \nu}=\zeta^{2}\left[v_{\gamma}^{-}\right]^{2}+\mu \zeta^{2} W_{2}+\nu \zeta W_{1}
\end{gathered}
$$

(notice that $\nu W_{1}$ is multiplied by $\zeta$ and not $\zeta^{2}$ ). Also set

$$
P_{\gamma \mu \nu} \phi=\zeta^{2} v_{\gamma}^{-} P_{\gamma} \phi+\mu \zeta^{2} v_{i j}^{-} \delta_{i} \delta_{j} \phi-\nu \zeta v_{i} \delta_{i} \phi .
$$

Observe that here the last term contains $\zeta$ to the first power unlike the other terms. Also note that

$$
P_{\gamma \mu \nu} v=-\zeta^{2}\left(v_{\gamma}^{-}\right)^{2}-\mu \zeta^{2} \sum_{i, j}\left|v_{i j}^{-}\right|^{2}-\nu \zeta W_{1}=-V_{\gamma \mu \nu} .
$$

A motivation for the chosen structure of $P_{\gamma \mu \nu}$ is given in Remarks 3.1 and 3.2 . The following result holds for any function $v$ not necessarily associated with (0.1).

Lemma 3.1. (i) For any $x$ and any $i, j$,

$$
\zeta\left|v_{i j}\right|(x) \leq \max _{x+\Lambda_{1}} \zeta W_{2}^{1 / 2}+N\left(d_{1}\right)\left\|\eta^{\prime}\right\| \max _{x+\Lambda_{2}} W_{1}^{1 / 2} .
$$

(ii) If

$$
3 \mu \max _{Q} \zeta^{2} W_{2} \leq \max _{Q} \zeta^{2}\left(v_{\gamma}^{-}\right)^{2}
$$

(which is equivalent to (2.2) ) and

$$
\nu \max _{Q} W_{1} \leq \mu \max _{Q} \zeta^{2} W_{2}
$$

then at any $x_{0} \in Q$ maximizing $V_{\gamma \mu \nu}$ we have

$$
\mu \max _{Q} \zeta^{2} W_{2} \leq \zeta^{2}\left[v_{\gamma}^{-}\right]^{2}, \quad \nu \max _{Q} W_{1} \leq \zeta^{2}\left[v_{\gamma}^{-}\right]^{2} .
$$

Furthermore, if additionally, $\mu$ satisfies (2.1) and

$$
h \sqrt{\nu} \leq \varepsilon
$$

then the operator $P_{\gamma \mu \nu}$ respects the maximum principle at $x_{0}$ relative to $\Lambda_{2}$, that is, for any function $\phi$ such that $\phi\left(x_{0}\right) \geq \phi\left(x_{0}+\eta\right)$ for all $\eta \in \Lambda_{2}$, we have $P_{\gamma \mu \nu} \phi\left(x_{0}\right) \leq$ 0 .

Proof. (i) Obviously $v_{-i, j}^{-} \leq W_{2}^{1 / 2}$ on $Q$. Since (no summation in $i$ ) $T_{h, l_{i}} v_{-i, j}^{-}=v_{i j}^{+}$, we get $v_{i j}^{+}(x) \leq W_{2}^{1 / 2}\left(x+h l_{i}\right)$ and

$$
\zeta(x) v_{i j}^{+}(x) \leq \zeta\left(x+h l_{i}\right) W_{2}^{1 / 2}\left(x+h l_{i}\right)+\left[\zeta(x)-\zeta\left(x+h l_{i}\right)\right] W_{2}^{1 / 2}\left(x+h l_{i}\right),
$$

which after taking into account the fact that $\left|\zeta(x)-\zeta\left(x+h l_{i}\right)\right| \leq 2 \| \eta^{\prime}|| h$ and that

$$
h W_{2}^{1 / 2}\left(x+h l_{i}\right) \leq N \max _{x+h l_{i}+\Lambda_{1}} W_{1}^{1 / 2}
$$

proves (i).

(ii) The second estimate in (3.5) follows from the first one and (3.4). Assuming that the first estimate in (3.5) does not hold, we obtain at $x_{0}$, 


$$
\begin{gathered}
V_{\gamma \mu \nu}<2 \mu \max _{Q} \zeta^{2} W_{2}+\nu \max _{Q} W_{1} \leq 3 \mu \max _{Q} \zeta^{2} W_{2}, \\
\max _{Q} \zeta^{2}\left[v_{\gamma}^{-}\right]^{2} \leq V_{\gamma \mu \nu}<3 \mu \max _{Q} \zeta^{2} W_{2}
\end{gathered}
$$

contrary to (3.3). This proves (3.5).

To prove the last assertion of the lemma we take a function with described properties and without loss of generality assume that $\phi\left(x_{0}\right)=0$. We also note that

$$
\begin{gathered}
h^{2} \delta_{i} \delta_{j} \phi\left(x_{0}\right)=\phi\left(x_{0}+h l_{i}+h l_{j}\right)-\phi\left(x_{0}+h l_{i}\right)-\phi\left(x_{0}+h l_{j}\right) \\
+\phi\left(x_{0}\right) \leq-\phi\left(x_{0}+h l_{i}\right)-\phi\left(x_{0}+h l_{j}\right)
\end{gathered}
$$

and $\Delta_{i} \phi\left(x_{0}\right) \leq 0$. Therefore, we infer from (3.5) that

$$
\begin{gathered}
h^{2} P_{\gamma \mu \nu} \phi\left(x_{0}\right) \leq \zeta^{2} v_{\gamma}^{-} \varepsilon \sum_{i}\left(\phi\left(x_{0}+h l_{i}\right)+\phi\left(x_{0}-h l_{i}\right)\right) \\
-\mu \zeta^{2} v_{i j}^{-}\left(\phi\left(x_{0}+h l_{i}\right)+\phi\left(x_{0}+h l_{j}\right)\right)-h \nu \zeta v_{i} \phi\left(x_{0}+h l_{i}\right) \\
\leq \zeta^{2} v_{\gamma}^{-}\left[\varepsilon \sum_{i}\left(\phi\left(x_{0}+h l_{i}\right)+\phi\left(x_{0}-h l_{i}\right)\right)\right. \\
\left.-\sqrt{\mu} \sum_{i, j}\left(\phi\left(x_{0}+h l_{i}\right)+\phi\left(x_{0}+h l_{j}\right)\right)-h \sqrt{\nu} \sum_{i} \phi\left(x_{0}+h l_{i}\right)\right] \\
=\zeta^{2} v_{\gamma}^{-} \sum_{i} \phi\left(x_{0}+h l_{i}\right)\left[2 \varepsilon-2 d_{1} \sqrt{\mu}-h \sqrt{\nu}\right] .
\end{gathered}
$$

The last expression is less than zero in light of the fact that $4 d_{1} \sqrt{\mu} \leq \varepsilon$ (see (2.1)) and $h \sqrt{\nu} \leq \varepsilon$. The lemma is proved.

Remark 3.1. Introducing $P_{\gamma \mu \nu}$ with $\zeta$ in front of the first-order differences may look strange. The reason for doing so is prompted by the desire to construct $P_{\gamma \mu \nu}$ satisfying the maximum principle.

Remark 3.2. Now we can explain how the operator $P_{\gamma \mu \nu}$ will be used. Assume (3.3), (3.4), (3.6), let $v$ satisfy (0.1) in $Q^{\circ}$ and (1.6) in $\partial Q$, and let $0 \in Q^{\circ}$. According to Remark 3.1 of 15 we may assume that the sup in (0.1) for $x=0$ is attained at $\alpha=\beta \in A$. Then the function

$$
U(x):=a_{k}^{\beta}(x) \Delta_{k} v(x)+b_{k}^{\beta}(x) \delta_{k} v(x)-c^{\beta}(x) v(x)+f^{\beta}(x)
$$

attains its maximum value over $\Lambda_{2}$ at 0 (note that $\Lambda_{2} \subset Q$ and if $\Lambda_{2} \backslash Q^{o}$ is nonempty, then this set is a subset of $\partial Q$ and $U \leq 0$ on this set owing to (1.6) ). By Lemma 3.1 we have $P_{\gamma \mu \nu} U \leq 0$ at 0 . While computing $P_{\gamma \mu \nu} U$ at 0 we find

$$
0 \geq\left[a_{k}^{\beta} \Delta_{k}+b_{k}^{\beta} \delta_{k}\right] P_{\gamma \mu \nu} v+2 \nu \zeta a_{k}^{\beta} \sum_{i} v_{i k}^{2}+\ldots
$$

By using (3.1) we obtain

$$
0 \geq-\left[a_{k}^{\beta} \Delta_{k}+b_{k}^{\beta} \delta_{k}\right] V_{\gamma \mu \nu}+2 \nu \zeta a_{k}^{\beta} \sum_{i} v_{i k}^{2}+\ldots
$$

If we additionally assume that at 0 the function $V_{\gamma \mu \nu}$ attains its maximum over $Q$, then at 0 ,

$$
\left[a_{k}^{\beta} \Delta_{k}+b_{k}^{\beta} \delta_{k}\right] V_{\gamma \mu \nu} \leq 0
$$

and (3.7) yields

$$
0 \geq 2 \nu \zeta a_{k}^{\beta} \sum_{i} v_{i k}^{2}+\ldots
$$


It turns out that here the terms expressed with ... are basically dominated by the term written explicitly and by some quantities related to $f$. This yields an estimate of $\zeta^{2} v_{i k}^{2}$ at 0 provided that the above additional assumptions are satisfied, which turns out to be the main case.

It turns out more convenient to start with (3.8) and extract some consequences without using the conditions (3.3), (3.4), (3.6), which will only play role in the main body of the proof of Theorem 2.1. Furthermore, in the following lemma, which we prove in Section 5, we are not going to use even the fact that $v$ satisfies (0.1) in $Q^{o}$ and (1.6) in $\partial Q$.

The following lemma allows us to estimate $P_{\gamma \mu \nu} U$ from below given that (3.8) holds. In Lemma 3.2 by $N$ we denote generic constants depending only on $\varepsilon, K, d_{1}$, and $\delta$ and by $N^{*}$ we denote those of $N$ 's which vanish if $a$ is independent of $x$ and $b \equiv c \equiv 0$.

Lemma 3.2. Let $\varepsilon, \mu \in(0,1], \nu \in(0, \infty), \gamma \in \Gamma(\varepsilon)$, and assume that

$$
V_{\gamma \mu \nu}(0) \geq V_{\gamma \mu \nu}\left(h l_{k}\right) \quad \forall k= \pm 1, \ldots, \pm d_{1} .
$$

There exist constants $N \geq 1$ and $N^{*} \geq 0$ such that if $h \leq h^{*}$, where $h^{*} \in(0,1]$ is any constant satisfying $N^{*} h^{*} \leq 1$, and

$$
\nu \geq N\left(\left\|\eta^{\prime \prime}\right\|+\left\|\eta^{\prime}\right\|^{2}\right)+N^{*} \mu^{-1}
$$

then, for any $\alpha \in A$, at point 0 we have

$$
\begin{gathered}
16 P_{\gamma \mu \nu}\left(a_{k}^{\alpha} \Delta_{k} v+b_{k}^{\alpha} \delta_{k} v\right) \\
\geq R^{\alpha \gamma}+\mu R^{\alpha \mu}+\nu R^{\alpha \nu}-\left(N^{*}+N \nu^{2}\right) \hat{W}_{1},
\end{gathered}
$$

where

$$
\begin{gathered}
\hat{W}_{1}=\max _{\Lambda_{2}} W_{1}, \quad R^{\alpha \gamma}=a_{k}^{\alpha} R_{k}^{\gamma}, \quad R^{\alpha \mu}=a_{k}^{\alpha} R_{k}^{\mu}, \quad R^{\alpha \nu}=a_{k}^{\alpha} R_{k}^{\nu}, \\
R_{k}^{\gamma}:=\left[\delta_{k}\left(\zeta v_{\gamma}^{-}\right)\right]^{2}, \quad R_{k}^{\mu}:=\sum_{i, j}\left[\delta_{k}\left(\zeta v_{i j}^{-}\right)\right]^{2}, \quad R_{k}^{\nu}:=\zeta \sum_{i} v_{k i}^{2} .
\end{gathered}
$$

Proof of Theorem 2.1. Take a constant $\nu$ satisfying (3.10) (that is, (2.3), recall that $\mu$ is fixed by (2.1) ) and introduce $x_{0}$ as a point in $Q$, where $V_{\gamma \mu \nu}$ attains its maximum. Without loss of generality we may assume that $x_{0}=0$. Next assume that $0 \in \partial Q$. Then at 0 ,

$$
\mu^{1 / 2} \max _{Q} \zeta W_{2}^{1 / 2} \leq V_{\gamma \mu \nu}^{1 / 2} \leq N\left(\max _{\partial Q, i, j}\left|\zeta v_{i j}\right|+\nu^{1 / 2} \max _{\partial Q, i}\left|\eta v_{i}\right|\right),
$$

which by (3.2) yields

$$
\max _{Q^{o}, i, j}\left|\zeta v_{i j}\right| \leq N \max _{\partial Q, i, j}\left|\zeta v_{i j}\right|+N\left(\nu^{1 / 2}+\left\|\eta^{\prime}\right\|\right) \max _{Q} W_{1}^{1 / 2} .
$$

After that (2.4) is immediate.

Therefore, in the rest of the proof we assume that

$$
x_{0}=0 \in Q^{o} .
$$

If (3.4) is violated, then

$$
\mu^{1 / 2} \max _{Q} \zeta W_{2}^{1 / 2} \leq \nu^{1 / 2} \max _{Q} W_{1}^{1 / 2}
$$

and (2.4) holds again. 
Hence, we may assume that (3.4) holds. We may also assume that $h$ satisfying $h \leq h_{0}$ also satisfies $h \leq h^{*}$, where $h^{*}$ is taken from Lemma 3.2. Indeed, if $h^{*} \leq h$, then

$$
\left|\zeta v_{i j}\right| \leq 2\left(h^{*}\right)^{-1} \max _{Q}\left|v_{i}\right|=N^{*} \max _{Q}\left|v_{i}\right| .
$$

Finally, we may assume that $h \sqrt{\nu} \leq \varepsilon$. Indeed, if $h \geq \varepsilon \nu^{-1 / 2}$, then in $Q^{o}$

$$
\left|\zeta v_{i j}\right| \leq 2 \nu^{1 / 2} \varepsilon^{-1} \max _{Q}\left|v_{i}\right| .
$$

After justifying these additional assumptions we may use the assertions of Lemmas 3.1 and 3.2. Furthermore, according to Remark 3.1 of 15 we may assume that there is an $\alpha \in A$ such that at 0 we have

$$
U:=a_{k}^{\alpha} \Delta_{k} v+b_{k}^{\alpha} \delta_{k} v-c^{\alpha} v+f^{\alpha}=0 .
$$

Also, obviously, $U(x) \leq 0$ for $x \in \Lambda_{2}$ (because $\Lambda_{2} \subset Q$ and (0.1) holds in $Q^{o}$ and (1.6) holds in $\partial Q$ ). Hence $U$ has a local maximum at point 0 . Since (3.3) and (3.4) are valid and $h \sqrt{\nu} \leq \varepsilon$, by Lemma 3.1 at $x_{0}$ we obtain

$$
P_{\gamma \mu \nu}\left(a_{k} \Delta_{k} v+b_{k} \delta_{k} v-c v+f\right) \leq 0,
$$

where and below we drop for brevity the superscript $\alpha$ and the argument $x=0$.

By Lemma 3.2

$$
0 \geq \nu R^{\nu}-\left(N^{*}+N \nu^{2}\right) \bar{W}_{1}+16 P_{\gamma \mu \nu}(f-c v) .
$$

Furthermore, by using simple manipulations (see, however, Lemma 4.1) and (3.1) we find that

$$
P_{\gamma \mu \nu}(c v)=-c V_{\gamma \mu \nu}+I
$$

where

$$
\begin{gathered}
I=v\left(\zeta^{2} v_{\gamma}^{-} \gamma_{k} \Delta_{k} c+\mu \zeta^{2} v_{i j}^{-} \delta_{i} \delta_{j} c-\nu \zeta v_{i} \delta_{i} c\right) \\
+2 \zeta^{2} v_{\gamma}^{-} \gamma_{k} v_{k} \delta_{k} c+2 \mu \zeta^{2} v_{i j}^{-} v_{i} \delta_{j} c \\
+2 h \mu \zeta^{2} v_{i j}^{-} v_{i j} \delta_{i} c+\mu \zeta^{2} v_{i j}^{-}\left(T_{l_{i}+l_{j}} v\right) \delta_{i} \delta_{j} c-h \nu \zeta v_{i} v_{i} \delta_{i} c .
\end{gathered}
$$
that

We use the fact that $h\left|v_{i j}\right| \leq N \bar{W}_{1}^{1 / 2}$ and $h\left|v_{i}\right| \leq N \bar{W}_{0}^{1 / 2}$ and easily conclude

$$
|I| \leq(1 / 64) \nu R^{\nu}+N^{*}\left(\nu+\nu^{-1}\right) \bar{W}_{0}+N^{*}\left(\nu+\nu^{-1}\right) \bar{W}_{1} .
$$

Moreover, obviously,

$$
P_{\gamma \mu \nu} f \geq-(1 / 64) \nu R^{\nu}-N \nu^{2} \bar{W}_{1}-N\left(\nu^{-1} K_{2 f}^{2}+K_{1 f}^{2}\right) .
$$

We also take into account that $c \geq 0$ and $V_{\gamma \mu \nu} \geq 0$ and then after coming back to (3.12) we conclude that at $x_{0}=0$,

$$
\begin{aligned}
\nu R^{\nu} \leq & N\left(\nu^{-1} K_{2 f}^{2}+K_{1 f}^{2}\right)+N^{*}\left(\nu+\nu^{-1}\right) \bar{W}_{0} \\
& +N^{*}\left(1+\nu^{-1}\right) \bar{W}_{1}+N \nu^{2} \bar{W}_{1} .
\end{aligned}
$$

Finally, observe that by (3.5) and (3.2)

$$
\mu \max _{Q} \zeta^{2} W_{2} \leq N R^{\nu}\left(x_{0}\right), \quad \max _{Q^{o}, i, j} \zeta^{2}\left|v_{i j}\right|^{2} \leq N R^{\nu}\left(x_{0}\right)+N\left\|\eta^{\prime}\right\|^{2} \bar{W}_{1} .
$$

The last estimate and (3.13) lead to (2.4) and the theorem is proved. 


\section{Some technicAl TOOLS}

Recall that the shift operator $T_{h, \xi}$ is introduced in (1.1).

Lemma 4.1. For any $i, j \in\left\{ \pm 1, \ldots, \pm d_{1}\right\}$ and functions $a(x), \psi(x)$

$$
\begin{gathered}
\delta_{i}(a \psi)=\left(\delta_{i} a\right) \psi+\left(T_{h, l_{i}} a\right) \delta_{i} \psi=a \delta_{i} \psi+\psi \delta_{i} a+h\left(\delta_{i} a\right) \delta_{i} \psi, \\
\delta_{i} \delta_{j}(a \psi)=a \delta_{i} \delta_{j} \psi+\left(\delta_{i} a\right) \delta_{j} \psi+\left(\delta_{j} a\right) \delta_{i} \psi \\
+h\left[\delta_{j} a+\delta_{i} a\right] \delta_{i} \delta_{j} \psi+\left(\delta_{i} \delta_{j} a\right) T_{h, l_{i}+l_{j}} \psi, \\
\Delta_{i}(a \psi)=a \Delta_{i} \psi+\psi \Delta_{i} a+\left(\delta_{i} a\right) \delta_{i} \psi+\left(\delta_{-i} a\right) \delta_{-i} \psi
\end{gathered}
$$

with no summation with respect to either $i$ or $j$. In particular,

$$
\Delta_{i}\left(\psi^{2}\right)=2 \psi \Delta_{i} \psi+\left(\delta_{i} \psi\right)^{2}+\left(\delta_{-i} \psi\right)^{2} .
$$

This lemma is Lemma 3.1 of [15] which is proved by straightforward computations. Before stating one more lemma we recall Definition 2.2 of [12].

Definition 4.1. Let $B$ be a finite subset of $\mathbb{R}^{d}, 0 \in B$, and let $p(x, y)$ be a realvalued function on $\mathbb{R}^{d} \times \mathbb{R}^{d}$. For an $x_{0} \in \mathbb{R}^{d}$ we say that the operator

$$
S: u \rightarrow S u, \quad S u(x)=\sum_{y \in B} p(x, x+y) u(x+y)
$$

respects the maximum principle at $x_{0}$ relative to $B$ if, for any function $\phi(x)$ such that $\phi\left(x_{0}+y\right) \geq \phi\left(x_{0}\right)$ for all $y \in B$, we have $S u\left(x_{0}\right) \geq 0$.

Lemma 4.2. If an operator

$$
S u(x)=\sum_{y \in B} p(x, x+y) u(x+y)
$$

respects the maximum principle at a point $x_{0} \in \mathbb{R}^{d}$ relative to $B$ and $u$ is a function such that $u\left(x_{0}\right) \leq 0$, then $-S u \leq S\left(u^{-}\right)$at $x_{0}$. In particular, $(S u)^{-} \leq\left(S\left(u^{-}\right)\right)^{+}$ and $\phi^{-} S \phi \geq-\phi^{-} S\left(\phi^{-}\right)$and $\phi^{-}(S \phi)^{-} \leq \phi^{-}\left|S\left(\phi^{-}\right)\right|$at $x_{0}$ for any function $\phi$.

This follows from the definition and the fact that $u+u^{-} \geq 0$ on $x_{0}+B$ and $u+u^{-}=0$ at $x_{0}$.

Recall that $h \leq h_{0} \leq 1$ and recall (1.3).

Lemma 4.3. For any $\alpha \in A$,

$$
a_{k}^{\alpha} \Delta_{k} \zeta=2 a_{k}^{\alpha} \Delta_{k} \eta+2 a_{k}^{\alpha}\left(\delta_{k} \eta\right)^{2}, \quad\left|\Delta_{k} \zeta\right| \leq 2\left(\left|\eta^{\prime \prime}\right|+\left|\eta^{\prime}\right|^{2}\right) .
$$

Furthermore, there is an absolute constant $N$ such that for any $k$ from $\left\{ \pm 1, \ldots, \pm d_{1}\right\}$,

$$
\delta_{k} \zeta=2 \eta \delta_{k} \eta+h\left(\delta_{k} \eta\right)^{2}, \quad\left(\delta_{k} \zeta\right)^{2} \leq N\left|\eta^{\prime}\right|^{2} \zeta+N h^{2}\left|\eta^{\prime}\right|^{4} \leq N\left|\eta^{\prime}\right|^{2},
$$

and for any $\chi \in(0,1]$, function $\psi$, and $i \in\left\{ \pm 1, \ldots, \pm d_{1}\right\}$,

$$
\begin{gathered}
h\left|\delta_{k} \zeta\right|^{2} \leq \chi \zeta+N \chi^{-1}\left|\eta^{\prime}\right|^{4} h^{2}, \\
\left|\left(\delta_{k} \zeta\right) \psi \delta_{i}(\zeta \psi)\right| \leq \chi\left|\delta_{i}(\zeta \psi)\right|^{2}+N \chi^{-1}\left|\eta^{\prime}\right|^{2}\left[\zeta \psi^{2}+\left|\eta^{\prime}\right|^{2}(h \psi)^{2}\right], \\
h\left(\delta_{k} \zeta\right)^{2}\left|\psi \delta_{i} \psi\right| \leq \chi\left|\delta_{i}(\zeta \psi)\right|^{2}+\chi\left|\eta^{\prime}\right|^{2} \zeta \psi^{2}+N \chi^{-1}\left|\eta^{\prime}\right|^{4}\left[(h \psi)^{2}+\left(h^{2} \delta_{i} \psi\right)^{2}\right], \\
\zeta^{3 / 2}\left|\delta_{i} \psi\right| \leq N\left|\delta_{i}(\zeta \psi)\right|+N\left|\eta^{\prime}\right| \zeta^{1 / 2}|\psi|+N\left|\eta^{\prime}\right|^{2}\left(h|\psi|+h^{2}\left|\delta_{i} \psi\right|\right) .
\end{gathered}
$$

This lemma is Lemma 3.3 of [15]. 


\section{Proof of Lemma 3.2}

The parameter $\alpha \in A$ is fixed throughout the section and we will drop this superscript in $a_{k}^{\alpha}, b_{k}^{\alpha}$, and $c_{k}^{\alpha}$. Recall that the constants $\varepsilon, \mu \in(0,1]$ and $\nu \in(0, \infty)$. We also suppose that $\gamma \in \Gamma(\varepsilon)$ and the remaining assumption (3.9) of Lemma 3.2 holds as well.

We introduce

$$
Z_{k}:=\zeta^{2} v_{\gamma}^{-} \Delta_{k} v_{\gamma}+\mu \zeta^{2} v_{i j}^{-} \Delta_{k} v_{i j}-\zeta \nu v_{i} \Delta_{k} v_{i}
$$

Observe that the term $a_{k}^{\alpha} Z_{k}$ arises as the "main" term in evaluation of the left-hand side of (3.8).

In this section by $N$ we denote generic constants depending only on $\varepsilon, K, d_{1}$, and $\delta$, unless specified explicitly otherwise, and by $N^{*}$ we denote those of $N^{\prime}$ 's which vanish if $a$ is independent of $x$ and $b \equiv c \equiv 0$.

Lemma 5.1. There exists a constant $N \geq 1$ such that if at the origin

$$
\nu \geq N\left(\left|\eta^{\prime \prime}\right|+\left|\eta^{\prime}\right|^{2}\right)
$$

then at 0 we have

$$
\begin{gathered}
2 a_{k} Z_{k} \geq-2 \zeta^{2} v_{\gamma}^{-} a_{k} \Delta_{k} v_{\gamma}^{-}-2 \mu \zeta^{2} v_{i j}^{-} a_{k} \Delta_{k} v_{i j}^{-}-2 \nu \zeta v_{i} a_{k} \Delta_{k} v_{i} \\
\geq-a_{k} \Delta_{k} V_{\gamma \mu \nu}+R^{\gamma}+\mu R^{\mu}+\nu R^{\nu}-N \nu^{2} \hat{W}_{1} \\
\geq R^{\gamma}+\mu R^{\mu}+\nu R^{\nu}-N \nu^{2} \hat{W}_{1} .
\end{gathered}
$$

Proof. The first inequality in (5.2) follows from Lemma 4.2, The last one is obtained on account of (3.9) since $\Delta_{k} V_{\gamma \mu \nu} \leq 0$ at the origin. To prove the remaining one we estimate $a_{k} \Delta_{k} V_{\gamma \mu \nu}$ from below at 0 . Observe that

$$
\begin{gathered}
a_{k} \Delta_{k}\left(\zeta^{2}\left(v_{\gamma}^{-}\right)^{2}\right)=2 \zeta v_{\gamma}^{-} a_{k} \Delta_{k}\left(\zeta v_{\gamma}^{-}\right)+2 a_{k}\left|\delta_{k}\left(\zeta v_{\gamma}^{-}\right)\right|^{2} \\
=2 \zeta^{2} v_{\gamma}^{-} a_{k} \Delta_{k} v_{\gamma}^{-}+2 \zeta\left(v_{\gamma}^{-}\right)^{2} a_{k} \Delta_{k} \zeta+4 \zeta v_{\gamma}^{-} a_{k}\left(\delta_{k} \zeta\right) \delta_{k} v_{\gamma}^{-}+2 a_{k}\left|\delta_{k}\left(\zeta v_{\gamma}^{-}\right)\right|^{2}
\end{gathered}
$$

and for each $k$,

$$
\zeta v_{\gamma}^{-}\left(\delta_{k} \zeta\right) \delta_{k} v_{\gamma}^{-}=v_{\gamma}^{-}\left(\delta_{k} \zeta\right) \delta_{k}\left(\zeta v_{\gamma}^{-}\right)-\left(v_{\gamma}^{-}\right)^{2}\left(\delta_{k} \zeta\right)^{2}-h v_{\gamma}^{-}\left(\delta_{k} \zeta\right)^{2} \delta_{k} v_{\gamma}^{-},
$$

so that

$$
\begin{gathered}
a_{k} \Delta_{k}\left(\zeta^{2}\left(v_{\gamma}^{-}\right)^{2}\right)=2 \zeta^{2} v_{\gamma}^{-} a_{k} \Delta_{k} v_{\gamma}^{-}+2 \zeta\left(v_{\gamma}^{-}\right)^{2} a_{k} \Delta_{k} \zeta+2 R^{\gamma} \\
+4 a_{k} v_{\gamma}^{-}\left(\delta_{k} \zeta\right) \delta_{k}\left(\zeta v_{\gamma}^{-}\right)-4\left(v_{\gamma}^{-}\right)^{2} a_{k}\left(\delta_{k} \zeta\right)^{2}-4 h v_{\gamma}^{-} a_{k}\left(\delta_{k} \zeta\right)^{2} \delta_{k} v_{\gamma}^{-} .
\end{gathered}
$$

Next,

$$
\begin{gathered}
a_{k} \Delta_{k}\left(\zeta^{2} W_{2}\right)=\sum_{i, j} a_{k} \Delta_{k}\left(\zeta v_{i j}^{-}\right)^{2}=2 a_{k} \zeta v_{i j}^{-} \Delta_{k}\left(\zeta v_{i j}^{-}\right)+2 \sum_{i, j} a_{k}\left|\delta_{k}\left(\zeta v_{i j}^{-}\right)\right|^{2} \\
=2 a_{k} \zeta^{2} v_{i j}^{-} \Delta_{k} v_{i j}^{-}+2 a_{k} \zeta W_{2} \Delta_{k} \zeta+4 a_{k} \zeta v_{i j}^{-}\left(\delta_{k} \zeta\right) \delta_{k} v_{i j}^{-}+2 R^{\mu}
\end{gathered}
$$

where for each $i, j, k$,

$$
\zeta v_{i j}^{-}\left(\delta_{k} \zeta\right) \delta_{k} v_{i j}^{-}=v_{i j}^{-}\left(\delta_{k} \zeta\right) \delta_{k}\left(\zeta v_{i j}^{-}\right)-\left(v_{i j}^{-}\right)^{2}\left(\delta_{k} \zeta\right)^{2}-h v_{i j}^{-}\left(\delta_{k} \zeta\right)^{2} \delta_{k} v_{i j}^{-}
$$

implying that

$$
\begin{gathered}
a_{k} \Delta_{k}\left(\zeta^{2} W_{2}\right)=2 a_{k} \zeta^{2} v_{i j}^{-} \Delta_{k} v_{i j}^{-}+2 a_{k} \zeta W_{2} \Delta_{k} \zeta+2 R^{\mu} \\
+4 a_{k} v_{i j}^{-}\left(\delta_{k} \zeta\right) \delta_{k}\left(\zeta v_{i j}^{-}\right)-4 a_{k} W_{2}\left(\delta_{k} \zeta\right)^{2}-4 h a_{k} v_{i j}^{-}\left(\delta_{k} \zeta\right)^{2} \delta_{k} v_{i j}^{-}
\end{gathered}
$$


Finally,

$$
\begin{gathered}
a_{k} \Delta_{k}\left(\zeta W_{1}\right)=\sum_{i} a_{k} \Delta_{k}\left(\zeta v_{i}^{2}\right)=\sum_{i} a_{k} \zeta\left[2 v_{i} \Delta_{k} v_{i}+2 v_{i k}^{2}\right] \\
+\sum_{i} a_{k} v_{i}^{2} \Delta_{k} \zeta+2 \sum_{i} a_{k}\left(\delta_{k} \zeta\right)\left[2 v_{i} \delta_{k} v_{i}+h v_{i k}^{2}\right] .
\end{gathered}
$$

We combine these computations and we see that at 0 ,

$$
\begin{aligned}
a_{k} \Delta_{k} V_{\gamma \mu \nu}=2 & a_{k}\left[\zeta^{2} v_{\gamma}^{-} \Delta_{k} v_{\gamma}^{-}+\mu \zeta^{2} v_{i j}^{-} \Delta_{k} v_{i j}^{-}+\nu \zeta v_{i} \Delta_{k} v_{i}\right] \\
& +2\left(R^{\gamma}+\mu R^{\mu}+\nu R^{\nu}\right)+I
\end{aligned}
$$

where $I=I_{1}+I_{2}+I_{3}+I_{4}$,

$$
\begin{gathered}
I_{1}=2\left[\zeta\left(v_{\gamma}^{-}\right)^{2}+\mu \zeta W_{2}+\nu W_{1}\right] a_{k} \Delta_{k} \zeta-4 a_{k}\left(\delta_{k} \zeta\right)^{2}\left[\left(v_{\gamma}^{-}\right)^{2}+\mu W_{2}\right]=: I_{11}+I_{12}, \\
I_{2}=4 a_{k}\left(\delta_{k} \zeta\right)\left[v_{\gamma}^{-} \delta_{k}\left(\zeta v_{\gamma}^{-}\right)+\mu v_{i j}^{-} \delta_{k}\left(\zeta v_{i j}^{-}\right)\right], \\
I_{3}=-4 h a_{k}\left(\delta_{k} \zeta\right)^{2}\left[v_{\gamma}^{-} \delta_{k} v_{\gamma}^{-}+\mu v_{i j}^{-} \delta_{k} v_{i j}^{-}\right], \\
I_{4}=2 \nu \sum_{i} a_{k}\left(\delta_{k} \zeta\right)\left[2 v_{i} \delta_{k} v_{i}+h v_{i k}^{2}\right] .
\end{gathered}
$$

It follows that to finish proving the lemma we need only prove that for appropriate values of $\nu$,

$$
|I| \leq R^{\gamma}+\mu R^{\mu}+\nu R^{\nu}+N \nu^{2} \hat{W}_{1} .
$$

We start with $I_{1}$. According to (4.3) (remember that $a_{k} \geq \delta$ )

$$
\left|I_{11}\right| \leq N\left(\left|\eta^{\prime \prime}\right|+\left|\eta^{\prime}\right|^{2}\right) R^{\nu}+N \nu\left(\left|\eta^{\prime \prime}\right|+\left|\eta^{\prime}\right|^{2}\right) \hat{W}_{1} .
$$

Also,

$$
\begin{aligned}
\left|I_{12}\right| & \leq N\left|\eta^{\prime}\right|^{2} \zeta\left[\left(v_{\gamma}^{-}\right)^{2}+W_{2}\right]+N\left|\eta^{\prime}\right|^{4} h^{2}\left[\left(v_{\gamma}^{-}\right)^{2}+W_{2}\right] \\
& \leq N\left|\eta^{\prime}\right|^{2} R^{\nu}+N\left|\eta^{\prime}\right|^{4}\left[\left(h v_{\gamma}^{-}\right)^{2}+\sum_{i, j}\left(h v_{i j}^{-}\right)^{2}\right] .
\end{aligned}
$$

Without any harm to some applications of the present lemma one could have assumed that $\zeta(0) \geq h^{2}$ and then one could estimate from above the last term in (5.5) by $N\left|\eta^{\prime}\right|^{4} R^{\nu}$. However, in the statement of the lemma this would lead to the requirement that $\nu \geq N\left|\eta^{\prime}\right|^{4}$ and this would lead to the fact that in the estimate of $\left|I_{11}\right|$ the coefficient of $\hat{W}_{1}$ would be larger than $N\left|\eta^{\prime}\right|^{6}$, which would ruin any hope of getting the correct rate of blow up of the second-order differences near the boundary.

Therefore, we use a different method. Observe that

$$
\left|h v_{i j}^{-}\right| \leq\left|h \delta_{i} \delta_{j} v\right|=\left|\left(T_{h, l_{i}}-1\right) \delta_{j} v\right| \leq 2 \hat{W}_{1}^{1 / 2},
$$

which implies that

$$
\begin{gathered}
\left|I_{12}\right| \leq N\left|\eta^{\prime}\right|^{2} R^{\nu}+N\left|\eta^{\prime}\right|^{4} \hat{W}_{1} \\
\left|I_{1}\right| \leq N\left(\left|\eta^{\prime \prime}\right|+\left|\eta^{\prime}\right|^{2}\right) R^{\nu}+N\left(\nu\left|\eta^{\prime \prime}\right|+\nu\left|\eta^{\prime}\right|^{2}+\left|\eta^{\prime}\right|^{4}\right) \hat{W}_{1} .
\end{gathered}
$$

While estimating $I_{2}$ we use (5.6) and (4.6) by taking first $\psi=v_{\gamma}$ and then $\psi=v_{i j}^{-}$. Then we find

$$
\left|I_{2}\right| \leq(1 / 2)\left(R^{\gamma}+\mu R^{\mu}\right)+N\left|\eta^{\prime}\right|^{2} R^{\nu}+N\left|\eta^{\prime}\right|^{4} \hat{W}_{1} .
$$


To estimate $I_{3}$ note that for any function $\phi$,

$$
\left|\delta_{k} \phi^{-}\right| \leq\left|\delta_{k} \phi\right|
$$

Also use (4.7) and (5.6). Then we find

$$
\left|I_{3}\right| \leq(1 / 4)\left(R^{\gamma}+\mu R^{\mu}\right)+N\left|\eta^{\prime}\right|^{2} R^{\nu}+N\left|\eta^{\prime}\right|^{4} \bar{W}_{1}+J,
$$

where

$$
J=N\left|\eta^{\prime}\right|^{4} \max _{i, j, k}\left|h^{2} \delta_{k} v_{i j}\right|^{2} .
$$

By observing that (here we use to the full extent the fact that $\hat{W}_{1}$ is defined as the maximum of $W_{1}$ over $\left.\Lambda_{2}\right)$

$$
\left|h^{2} \delta_{k} v_{i j}\right|=\left|\left(T_{h, l_{i}}-1\right)\left(T_{h, l_{j}}-1\right) \delta_{k} v\right| \leq N \hat{W}_{1}^{1 / 2},
$$

we see that $J \leq N\left|\eta^{\prime}\right|^{4} \hat{W}_{1}$ and

$$
\left|I_{3}\right| \leq(1 / 4)\left(R^{\gamma}+\mu R^{\mu}\right)+N\left|\eta^{\prime}\right|^{2} R^{\nu}+N\left|\eta^{\prime}\right|^{4} \hat{W}_{1} .
$$

Turning our attention to $I_{4}$, owing to (5.6), we get

$$
\begin{gathered}
\left|I_{4}\right| \leq N \nu \sum_{i} a_{k}\left(\left|\eta^{\prime}\right| \sqrt{\zeta}+h\left|\eta^{\prime}\right|^{2}\right)\left|2 v_{i} v_{i k}+h v_{i k}^{2}\right| \\
\leq N \nu \hat{W}_{1}^{1 / 2} \sum_{i} a_{k}\left(\left|\eta^{\prime}\right| \sqrt{\zeta}+h\left|\eta^{\prime}\right|^{2}\right)\left|v_{i k}\right| \leq N \nu\left|\eta^{\prime}\right| \hat{W}_{1}^{1 / 2}\left(R^{\nu}\right)^{1 / 2} \\
+N \nu\left|\eta^{\prime}\right|^{2} \hat{W}_{1} \leq(1 / 2) \nu R^{\nu}+N \nu\left|\eta^{\prime}\right|^{2} \hat{W}_{1} .
\end{gathered}
$$

Upon combining this result with (5.7), (5.8), and (5.11) we conclude that

$$
\begin{gathered}
|I| \leq\left|I_{1}\right|+\left|I_{2}\right|+\left|I_{3}\right|+\left|I_{4}\right| \leq N_{1}\left[\nu\left|\eta^{\prime \prime}\right|+\nu\left|\eta^{\prime}\right|^{2}+\left|\eta^{\prime}\right|^{4}\right] \hat{W}_{1} \\
+R^{\gamma}+\mu R^{\mu}+N_{2}\left(\left|\eta^{\prime \prime}\right|+\left|\eta^{\prime}\right|^{2}\right) R^{\nu}+(1 / 2) \nu R^{\nu},
\end{gathered}
$$

where we may safely assume that $N_{2} \geq 1$. By choosing $\nu$ so that

$$
N_{2}\left(\left|\eta^{\prime \prime}\right|+\left|\eta^{\prime}\right|^{2}\right) \leq \nu / 2
$$

and noting that then $\left|\eta^{\prime}\right|^{2},\left|\eta^{\prime \prime}\right| \leq \nu$ and

$$
N_{1}\left(\nu\left|\eta^{\prime \prime}\right|+\nu\left|\eta^{\prime}\right|^{2}+(1+\mu)\left|\eta^{\prime}\right|^{4}\right) \leq N \nu^{2},
$$

we arrive at (5.4) and the lemma is proved.

Set

$$
z_{k}:=\zeta^{2} v_{\gamma}^{-} \delta_{k} v_{\gamma}+\mu \zeta^{2} v_{i j}^{-} \delta_{k} v_{i j}-\nu \zeta v_{i} v_{k i} .
$$

Lemma 5.2. There exist constants $N \geq 1$ and $N^{*} \geq 0$ such that if at the origin

$$
\nu \geq N\left(\left|\eta^{\prime \prime}\right|+\left|\eta^{\prime}\right|^{2}\right)+N^{*}\left|\eta^{\prime}\right|\left(1+\nu^{-1 / 2}\right),
$$

then at 0 we have

$$
16\left(a_{k} Z_{k}+b_{k} z_{k}\right) \geq 4 a_{k} Z_{k}+R^{\gamma}+\mu R^{\mu}+\nu R^{\nu}-N \nu^{2} \hat{W}_{1} .
$$


Proof. Observe that since $a_{k} \geq 2\left(b_{k}\right)^{-}$, the operator $a_{k} \Delta_{k}+2 b_{k} \delta_{k}$ respects the maximum principle, so that by Lemma 4.2 and (5.3) we have

$$
\begin{gathered}
0 \geq\left(a_{k} \Delta_{k}+2 b_{k} \delta_{k}\right) V_{\gamma \mu \nu}=2\left[\zeta^{2} v_{\gamma}^{-}\left(a_{k} \Delta_{k}+2 b_{k} \delta_{k}\right) v_{\gamma}^{-}\right. \\
\left.+\mu \zeta^{2} v_{i j}^{-}\left(a_{k} \Delta_{k}+2 b_{k} \delta_{k}\right) v_{i j}^{-}+\nu \zeta v_{i}\left(a_{k} \Delta_{k}+2 b_{k} \delta_{k}\right) v_{i}\right] \\
+2\left(a_{k}+h b_{k}\right)\left(R_{k}^{\gamma}+\mu R_{k}^{\mu}+\nu R_{k}^{\nu}\right)+I+J,
\end{gathered}
$$

where $J=J_{1}+J_{2}+J_{3}$,

$$
\begin{gathered}
J_{1}=2 b_{k}\left(\delta_{k} \zeta\right)\left[2 \zeta\left(v_{\gamma}^{-}\right)^{2}+2 \mu \zeta W_{2}+\nu W_{1}\right], \\
J_{2}=2 \nu h b_{k}\left(\delta_{k} \zeta\right)\left[2 v_{i} v_{i k}+h \sum_{i} v_{i k}^{2}\right], \\
J_{3}=4 h \zeta b_{k}\left(\delta_{k} \zeta\right)\left[v_{\gamma}^{-} \delta_{k} v_{\gamma}^{-}+\mu v_{i j}^{-} \delta_{k} v_{i j}^{-}\right] .
\end{gathered}
$$

By Lemma 4.2,

$$
\begin{gathered}
v_{\gamma}^{-}\left(a_{k} \Delta_{k}+2 b_{k} \delta_{k}\right) v_{\gamma}^{-} \geq-v_{\gamma}^{-}\left(a_{k} \Delta_{k}+2 b_{k} \delta_{k}\right) v_{\gamma}, \\
v_{i j}^{-}\left(a_{k} \Delta_{k}+2 b_{k} \delta_{k}\right) v_{i j}^{-} \geq-v_{i j}^{-}\left(a_{k} \Delta_{k}+2 b_{k} \delta_{k}\right) v_{i j} .
\end{gathered}
$$

Furthermore, $a_{k}+h b_{k} \geq(1 / 2) a_{k}$. Therefore, recalling the definitions of $Z_{k}$ and $z_{k}$, we obtain from (5.15) that

$$
2 a_{k} Z_{k}+4 b_{k} z_{k} \geq R^{\gamma}+\mu R^{\mu}+\nu R^{\nu}+I+J
$$

which along with (5.2) and (5.4) yields

$$
\begin{gathered}
4 a_{k} Z_{k}+4 b_{k} z_{k} \geq a_{k} Z_{k}+(3 / 2)\left(R^{\gamma}+\mu R^{\mu}+\nu R^{\nu}\right)+I+J-N \nu^{2} \hat{W}_{1} \\
\geq a_{k} Z_{k}+(1 / 2)\left(R^{\gamma}+\mu R^{\mu}+\nu R^{\nu}\right)+J-N \nu^{2} \hat{W}_{1}
\end{gathered}
$$

provided that $\nu$ satisfies (5.1).

Next, obviously,

$$
\left|J_{1}\right| \leq N^{*}\left|\eta^{\prime}\right|(1+\mu) R^{\nu}+N^{*} \nu\left|\eta^{\prime}\right| W_{1} .
$$

To estimate $J_{2}$ we use (5.6) and easily find that

$$
J_{2} \leq N^{*} \nu\left|\eta^{\prime}\right| \hat{W}_{1} \text {. }
$$

To estimate $J_{3}$ observe that

$$
\begin{gathered}
\left|4 h \zeta b_{k}\left(\delta_{k} \zeta\right) v_{\gamma}^{-} \delta_{k} v_{\gamma}^{-}\right|=\left|4 h b_{k}\left(\delta_{k} \zeta\right) v_{\gamma}^{-}\left[\delta_{k}\left(\zeta v_{\gamma}^{-}\right)-\left(\delta_{k} \zeta\right) T_{h, l_{k}} v_{\gamma}^{-}\right]\right| \\
\leq N^{*}\left|\eta^{\prime}\right| \hat{W}_{1}^{1 / 2}\left(R^{\gamma}\right)^{1 / 2}+N^{*} v_{\gamma}^{-} \sum_{k}\left(\delta_{k} \zeta\right)^{2} \hat{W}_{1}^{1 / 2},
\end{gathered}
$$

where, owing to the fact that $h\left|\eta^{\prime}\right| \leq 2$, the last term is dominated by

It follows that

$$
\begin{gathered}
N^{*} \hat{W}_{1}^{1 / 2} v_{\gamma}^{-}\left(\zeta\left|\eta^{\prime}\right|^{2}+h^{2}\left|\eta^{\prime}\right|^{4}\right) \\
\leq\left(N^{*} \zeta^{1 / 2}\left|\eta^{\prime}\right|^{3 / 2} \hat{W}_{1}^{1 / 2}\right)\left(\left|\eta^{\prime}\right|^{1 / 2} \zeta^{1 / 2} v_{\gamma}^{-}\right)+N^{*}\left|\eta^{\prime}\right|^{3} \hat{W}_{1} .
\end{gathered}
$$

$$
\left|4 h \zeta b_{k}\left(\delta_{k} \zeta\right) v_{\gamma}^{-} \delta_{k} v_{\gamma}^{-}\right| \leq(1 / 4) R^{\gamma}+N^{*}\left|\eta^{\prime}\right| R^{\nu}+N^{*}\left(\left|\eta^{\prime}\right|^{2}+\left|\eta^{\prime}\right|^{3}\right) \hat{W}_{1} .
$$

Similarly, one estimates $\left|4 \mu h \zeta b_{k}\left(\delta_{k} \zeta\right) v_{i j}^{-} \delta_{k} v_{i j}^{-}\right|$and concludes that

$$
\begin{aligned}
& \left|J_{3}\right| \leq(1 / 4) R^{\gamma}+(1 / 4) \mu R^{\mu}+N^{*}\left|\eta^{\prime}\right| R^{\nu}+N^{*}\left(\left|\eta^{\prime}\right|^{2}+\left|\eta^{\prime}\right|^{3}\right) \hat{W}_{1} . \\
& |J| \leq(1 / 4) R^{\gamma}+(1 / 4) \mu R^{\mu}+N_{1}^{*}\left|\eta^{\prime}\right| R^{\nu}+N_{2}^{*}\left|\eta^{\prime}\right|\left(\left|\eta^{\prime}\right|+\left|\eta^{\prime}\right|^{2}+\nu\right) \hat{W}_{1} .
\end{aligned}
$$


Now, if $\nu$ not only satisfies (5.1) but is also such that

$$
\left(N_{1}^{*}+N_{2}^{*}\right)\left|\eta^{\prime}\right|\left(2+\nu^{-1 / 2}\right) \leq(1 / 2) \nu
$$

then

$$
N_{2}^{*}\left|\eta^{\prime}\right|\left(\left|\eta^{\prime}\right|+\left|\eta^{\prime}\right|^{2}+\nu\right) \leq N_{2}^{*}\left|\eta^{\prime}\right|\left(\nu^{1 / 2}+2 \nu\right) \leq \nu^{2}
$$

and (5.17) along with (5.16) yields (5.14) and proves the lemma.

The following lemma from [1] will be also used.

Lemma 5.3. Let $\psi$ be a function on $\mathbb{R}^{d}$. Then, for any $k \in\left\{ \pm 1, \ldots, \pm d_{1}\right\}$,

$$
\left|\Delta_{k} \psi\right| \leq\left|\delta_{-k}\left(\left(\delta_{k} \psi\right)^{-}\right)\right|+\left|\delta_{k}\left(\left(\delta_{-k} \psi\right)^{-}\right)\right| .
$$

Proof of Lemma 3.2. Set

$$
J:=P_{\gamma \mu \nu}\left(a_{k}^{\alpha} \Delta_{k} v+b_{k}^{\alpha} \delta_{k} v\right) .
$$

Borrowing the result of computations in the proof of Lemma 6.5 of [12] we see that at 0 we have

$$
J=a_{k} Z_{k}+b_{k} z_{k}+I_{1}+\cdots+I_{4},
$$

where

$$
\begin{gathered}
I_{1}=-2 \zeta^{2} \gamma_{0 i} v_{\gamma}^{-}\left(\delta_{-i} a_{k}\right) \Delta_{k} v_{i}+2 \mu \zeta^{2} v_{i j}^{-}\left(\delta_{j} a_{k}\right) \Delta_{k} v_{i} \\
I_{2}=\mu \zeta^{2} h v_{i j}^{-}\left[\left(\delta_{j}+\delta_{i}\right) a_{k}\right] \Delta_{k} v_{i j} \\
I_{3}=\zeta^{2} v_{\gamma}^{-}\left[4 \gamma_{0 i}\left(\Delta_{i} a_{k}\right) v_{k i}+\gamma_{0 i}\left(\Delta_{i} a_{k}\right) \Delta_{k} v\right] \\
+\mu \zeta^{2} v_{i j}^{-}\left(\delta_{j} \delta_{i} a_{k}\right) T_{h, l_{j}+l_{i}} \Delta_{k} v \\
+2 \mu \zeta^{2} v_{i j}^{-}\left(\delta_{j} b_{k}\right) v_{k i}+2 \mu \zeta^{2} h v_{i j}^{-}\left(\delta_{i} b_{k}\right) \delta_{k} v_{i j}+\mu \zeta^{2} v_{i j}^{-}\left(\delta_{j} \delta_{i} b_{k}\right) T_{h, l_{i}+l_{j}} v_{k} \\
-\nu \zeta v_{i}\left(\delta_{i} a_{k}\right)\left(\Delta_{k} v+2 v_{k i}\right)-\nu \zeta v_{i}\left(\delta_{i} b_{k}\right) T_{h, l_{i}} v_{k} .
\end{gathered}
$$

For $\nu$ satisfying

$$
\nu \geq N\left(\left\|\eta^{\prime \prime}\right\|+\left\|\eta^{\prime}\right\|^{2}\right)+N^{*}\left\|\eta^{\prime}\right\|\left(1+\nu^{-1 / 2}\right),
$$

with $N$ and $N^{*}$ from Lemma 5.2 (which we assume throughout the proof before a stronger assumption is imposed) it follows by Lemma 5.2 that

$$
16 J \geq 4 a_{k} Z_{k}+R^{\gamma}+\mu R^{\mu}+\nu R^{\nu}+16 I_{1}+\cdots+16 I_{4}-N \nu^{2} \hat{W}_{1} .
$$

Estimating $I_{1}$. Note that (recall that $\mu \leq 1$ )

$$
\left|16 I_{1}\right| \leq N^{*}\left(R^{\nu}\right)^{1 / 2} \zeta^{3 / 2} \sum_{i, k}\left|\Delta_{k} v_{i}\right|
$$

and by (5.18) (no summation in $k$ )

$$
\zeta^{3 / 2}\left|\Delta_{k} v_{i}\right| \leq \zeta^{1 / 2}\left[\left|\zeta \delta_{-k} v_{k i}^{-}\right|+\left|\zeta \delta_{k} v_{-k, i}^{-}\right|\right] .
$$

Observe that by (4.8) for any $i, j, k$,

$$
\zeta^{3 / 2}\left|\delta_{k} v_{i j}^{-}\right| \leq N\left|\delta_{k}\left(\zeta v_{i j}^{-}\right)\right|+N\left|\eta^{\prime}\right| \zeta^{1 / 2} v_{i j}^{-}+N\left|\eta^{\prime}\right|^{2}\left(h v_{i j}^{-}+h^{2}\left|\delta_{k} v_{i j}^{-}\right|\right)
$$

We estimate the last term by using (5.9), (5.6), and (5.10) and conclude that for any $i, j, k$,

$$
\zeta^{3 / 2}\left|\delta_{k} v_{i j}^{-}\right| \leq N\left(R^{\mu}\right)^{1 / 2}+N\left|\eta^{\prime}\right|\left(R^{\nu}\right)^{1 / 2}+N\left|\eta^{\prime}\right|^{2} \hat{W}_{1}^{1 / 2} .
$$

Then we find from (5.21) that

$$
\left|16 I_{1}\right| \leq(1 / 4) \mu R^{\mu}+N^{*}\left(\mu^{-1}+\left|\eta^{\prime}\right|\right) R^{\nu}+N\left|\eta^{\prime}\right|^{4} \hat{W}_{1} .
$$


Estimating $I_{2}$. This takes more time. We start by observing that

$$
\left|16 I_{2}\right| \leq N_{1}^{*} \mu \zeta^{2} h v_{i j}^{-} a_{k}\left|\Delta_{k} v_{i j}\right| .
$$

Furthermore, by the formula $|\theta|=\theta+2 \theta^{-}$, Lemma 4.2, and the fact that $h \Delta_{k}=$ $\delta_{k}+\delta_{-k}$ we have

$$
\begin{gathered}
\mu \zeta^{2} h v_{i j}^{-} a_{k}\left|\Delta_{k} v_{i j}\right|=\mu \zeta^{2} h v_{i j}^{-} a_{k} \Delta_{k} v_{i j}+2 \mu \zeta^{2} h v_{i j}^{-} a_{k}\left(\Delta_{k} v_{i j}\right)^{-} \\
\leq \mu \zeta^{2} h v_{i j}^{-} a_{k} \Delta_{k} v_{i j}+2 \mu \zeta^{2} h v_{i j}^{-} a_{k}\left|\Delta_{k} v_{i j}^{-}\right| \\
\leq \mu \zeta^{2} h v_{i j}^{-} a_{k} \Delta_{k} v_{i j}+4 \mu \zeta^{2} v_{i j}^{-} a_{k}\left|\delta_{k} v_{i j}^{-}\right| \\
=h a_{k} Z_{k}+I_{21}+I_{22}
\end{gathered}
$$

where

$$
I_{21}=-h a_{k}\left(\zeta^{2} v_{\gamma}^{-} \Delta_{k} v_{\gamma}-\zeta \nu v_{i} \Delta_{k} v_{i}\right), \quad I_{22}=4 \mu \zeta^{2} v_{i j}^{-} a_{k}\left|\delta_{k} v_{i j}^{-}\right| .
$$

By using (5.22) we find that

$$
I_{22} \leq N \mu\left(R^{\nu}\right)^{1 / 2} \zeta^{3 / 2} \sum_{i, j, k}\left|\delta_{k} v_{i j}^{-}\right|
$$

$$
\leq N \mu\left(R^{\nu}\right)^{1 / 2}\left[\left(R^{\mu}\right)^{1 / 2}+\left|\eta^{\prime}\right|\left(R^{\nu}\right)^{1 / 2}+\left|\eta^{\prime}\right|^{2} \hat{W}_{1}^{1 / 2}\right] .
$$

It follows that (we also use the fact that $\mu \leq 1$ )

$$
N_{1}^{*} I_{22} \leq(1 / 4) \mu R^{\mu}+N^{*}\left(1+\left|\eta^{\prime}\right|\right) R^{\nu}+N\left|\eta^{\prime}\right|^{4} \hat{W}_{1} .
$$

To estimate $I_{21}$ we use Lemma 4.2 to get

$$
\begin{gathered}
I_{21} \leq 2 a_{k}\left(\zeta^{2} v_{\gamma}^{-} \delta_{k} v_{\gamma}^{-}+\zeta \nu v_{i} v_{k i}\right) \\
\leq N\left(R^{\nu}\right)^{1 / 2}\left(\zeta^{3 / 2} \sum_{k}\left|\delta_{k} v_{\gamma}^{-}\right|+\nu \hat{W}_{1}^{1 / 2}\right),
\end{gathered}
$$

where by (4.8)

$$
\zeta^{3 / 2}\left|\delta_{k} v_{\gamma}^{-}\right| \leq N\left(R^{\gamma}\right)^{1 / 2}+N\left|\eta^{\prime}\right|\left(R^{\nu}\right)^{1 / 2}+N\left|\eta^{\prime}\right|^{2} \hat{W}_{1}^{1 / 2} .
$$

It follows that

$$
\begin{gathered}
\left|I_{21}\right| \leq N\left(R^{\nu}\right)^{1 / 2}\left[\left(R^{\gamma}\right)^{1 / 2}+\left|\eta^{\prime}\right|\left(R^{\nu}\right)^{1 / 2}+\left(\nu+\left|\eta^{\prime}\right|^{2}\right) \hat{W}_{1}^{1 / 2}\right] \\
N_{1}^{*} I_{21} \leq(1 / 4) R^{\gamma}+N^{*}\left(1+\left|\eta^{\prime}\right|\right) R^{\nu}+N\left(\nu^{2}+\left|\eta^{\prime}\right|^{4}\right) \hat{W}_{1} .
\end{gathered}
$$

Hence,

$$
\begin{gathered}
\left|16 I_{2}\right| \leq N_{1}^{*} h a_{k} Z_{k}+(1 / 4)\left(R^{\gamma}+\mu R^{\mu}\right) \\
+N^{*}\left(1+\left|\eta^{\prime}\right|\right) R^{\nu}+N\left(\nu^{2}+\left|\eta^{\prime}\right|^{4}\right) \hat{W}_{1} .
\end{gathered}
$$

Estimating $I_{3}$. We use the following result of simple computations:

$$
T_{h, l_{j}+l_{i}} \Delta_{k} v=-v_{k,-k}+v_{k j}+v_{-k, j}+v_{k i}+v_{-k, i}+h^{2} \Delta_{k} v_{i j} .
$$

This shows new terms entering $I_{3}$. All of them, apart from the last one, are similar to the ones which are written explicitly in the definition of $I_{3}$ and are obviously estimated from above by $N^{*} R^{\nu}$. To estimate the remaining term we use (5.25), (5.26), and (5.27) to obtain that

$$
\begin{gathered}
\mu \zeta^{2} h^{2} v_{i j}^{-} a_{k}\left|\Delta_{k} v_{i j}\right| \leq h^{2} a_{k} Z_{k} \\
+N h\left(R^{\nu}\right)^{1 / 2}\left[\left(R^{\gamma}\right)^{1 / 2}+\mu\left(R^{\mu}\right)^{1 / 2}+\left|\eta^{\prime}\right|\left(R^{\nu}\right)^{1 / 2}+\left(\nu+\left|\eta^{\prime}\right|^{2}\right) \hat{W}_{1}^{1 / 2}\right] .
\end{gathered}
$$


It follows that

$$
\begin{gathered}
\left|16 I_{3}\right| \leq N_{2}^{*} h^{2} a_{k} Z_{k}+(1 / 4)\left(R^{\gamma}+\mu R^{\mu}\right) \\
+N^{*}\left(1+\left|\eta^{\prime}\right|\right) R^{\nu}+N\left(\nu^{2}+\left|\eta^{\prime}\right|^{4}\right) \hat{W}_{1} .
\end{gathered}
$$

Estimating $I_{4}$. All terms entering $16 I_{4}$ apart from

$$
I_{41}=32 \mu \zeta^{2} h v_{i j}^{-}\left(\delta_{i} b_{k}\right) \delta_{k} v_{i j}
$$

are obviously dominated by

$$
N^{*} R^{\nu}+(1 / 4) \nu R^{\nu}+N^{*}(1+\nu) \hat{W}_{1} .
$$

Furthermore,

$$
\left|I_{41}\right| \leq N^{*} h \mu \zeta^{2} v_{i j}^{-} a_{k}\left|\delta_{k} v_{i j}\right|
$$

where

$$
\mu \zeta^{2} v_{i j}^{-} a_{k}\left|\delta_{k} v_{i j}\right|=\mu \zeta^{2} v_{i j}^{-} a_{k} \delta_{k} v_{i j}+2 \mu \zeta^{2} v_{i j}^{-} a_{k}\left(\delta_{k} v_{i j}\right)^{-},
$$

which by Lemma 4.2 is less than

$$
(1 / 2) \mu \zeta^{2} h v_{i j}^{-} a_{k} \Delta_{k} v_{i j}+(1 / 2) I_{22}=(1 / 2)\left[h a_{k} Z_{k}+I_{21}+I_{22}\right] .
$$

By using (5.26) and (5.27) we estimate the last expression by

It follows that

$$
\begin{gathered}
(1 / 2) h a_{k} Z_{k}+N\left(R^{\nu}\right)^{1 / 2}\left[\left(R^{\gamma}\right)^{1 / 2}\right. \\
\left.+\mu\left(R^{\mu}\right)^{1 / 2}+\left|\eta^{\prime}\right|\left(R^{\nu}\right)^{1 / 2}+\left(\nu+\left|\eta^{\prime}\right|^{2}\right) \hat{W}_{1}^{1 / 2}\right] .
\end{gathered}
$$

$$
\begin{gathered}
\left|I_{41}\right| \leq N_{3}^{*} h^{2} a_{k} Z_{k} \\
+N^{*} h\left(R^{\nu}\right)^{1 / 2}\left[\left(R^{\gamma}\right)^{1 / 2}+\mu\left(R^{\mu}\right)^{1 / 2}+\left|\eta^{\prime}\right|\left(R^{\nu}\right)^{1 / 2}+\left(\nu+\left|\eta^{\prime}\right|^{2}\right) \hat{W}_{1}^{1 / 2}\right] .
\end{gathered}
$$

Next we use the fact that $h\left|\eta^{\prime}\right| \leq 2$ and $h^{2} R^{\nu} \leq N \hat{W}_{1}$. Then we obtain

$$
\begin{gathered}
\left|I_{41}\right| \leq N_{3}^{*} h^{2} a_{k} Z_{k}+(1 / 4)\left(R^{\gamma}+\mu R^{\mu}\right) \\
+N^{*} R^{\nu}+N^{*}\left(1+\nu+\left|\eta^{\prime}\right|^{2}\right) \hat{W}_{1} .
\end{gathered}
$$

Now we collect the above estimates of parts of $\left|16 I_{4}\right|$ and use simple inequalities like

$$
\nu \leq 1+\nu^{2}, \quad\left|\eta^{\prime}\right|^{2} \leq 1+\left|\eta^{\prime}\right|^{4}
$$

These rough estimates will make the estimate of $\left|16 I_{4}\right|$ rougher, but we had the terms appearing in this estimate in the estimates of $\left|16 I_{i}\right|, i \leq 4$, anyway. We get that

$$
\begin{gathered}
\left|16 I_{4}\right| \leq N_{3}^{*} h^{2} a_{k} Z_{k}+(1 / 4)\left(R^{\gamma}+\mu R^{\mu}+\nu R^{\nu}\right)+N^{*} R^{\nu} \\
+N\left(N^{*}+\nu^{2}+\left|\eta^{\prime}\right|^{4}\right) \hat{W}_{1} .
\end{gathered}
$$

By combining this with (5.23), (5.28), and (5.29), we see that

$$
\begin{gathered}
16\left(\left|I_{1}\right|+\left|I_{2}\right|+\left|I_{3}\right|+\left|I_{4}\right|\right) \leq\left(N_{1}^{*} h+N_{2}^{*} h^{2}+N_{3}^{*} h^{2}\right) a_{k} Z_{k} \\
+R^{\gamma}+\mu R^{\mu}+\nu R^{\nu}+N^{*}\left(\mu^{-1}+\left|\eta^{\prime}\right|\right) R^{\nu} \\
-(1 / 2) \nu R^{\nu}+N\left(N^{*}+\nu^{2}+\left|\eta^{\prime}\right|^{4}\right) \hat{W}_{1} .
\end{gathered}
$$

We choose $h^{*} \in(0,1]$ so that

$$
\left(N_{1}^{*}+N_{2}^{*}+N_{3}^{*}\right) h^{*} \leq 2,
$$

and use the fact that for $h \leq h^{*}$ we have

$$
2 \geq 2-\left(N_{1}^{*} h+N_{2}^{*} h^{2}+N_{3}^{*} h^{2}\right) \geq 0 .
$$


Then we take into account that owing to (5.2) we have

$$
4 a_{k} Z_{k}-\left(N_{1}^{*} h+N_{2}^{*} h^{2}+N_{3}^{*} h^{2}\right) a_{k} Z_{k} \geq R^{\gamma}+\mu R^{\mu}+\nu R^{\nu}-N \nu^{2} \hat{W}_{1} .
$$

After that we come back to (5.20) and obtain that

$$
\begin{gathered}
16 J \geq R^{\gamma}+\mu R^{\mu}+\nu R^{\nu} \\
-N_{4}^{*}\left(\mu^{-1}+\left|\eta^{\prime}\right|\right) R^{\nu}+(1 / 2) \nu R^{\nu}-N_{1}\left(N_{5}^{*}+\nu^{2}+\left|\eta^{\prime}\right|^{4}\right) \hat{W}_{1} .
\end{gathered}
$$

We now require that $\nu$ not only satisfy (5.19) but also be such that

$$
\nu / 2 \geq N_{4}^{*}\left(\mu^{-1}+\left|\eta^{\prime}\right|\right) .
$$

Then

$$
N_{1}\left(N_{5}^{*}+\nu^{2}+\left|\eta^{\prime}\right|^{4}\right) \leq N\left(N^{*}+\nu^{2}\right) \hat{W}_{1}
$$

and (5.30) yields (3.11). After that it only remains to observe that condition (3.10) with appropriate $N, N^{*}$ implies both (5.19) and (5.31). Indeed, if the constants $N^{*}$ vanish, there is nothing to prove. If they are not, we choose $N^{*}$ in (3.10) to be at least bigger than one. Then automatically $\nu \geq 1$ and, since $\left|\eta^{\prime}\right|^{2} \leq \nu$,

$$
N^{*}\left|\eta^{\prime}\right|\left(1+\nu^{-1 / 2}\right) \leq N^{*}+(1 / 2) \nu \leq N^{*} \mu^{-1}+(1 / 2) \nu .
$$

A similar argument shows that on account of increasing $N^{*}$ in (3.10) one can satisfy (5.31) as well, which brings the proof of the lemma to an end.

\section{REFERENCES}

[1] G. Barles and E.R. Jakobsen, Error bounds for monotone approximation schemes for parabolic Hamilton-Jacobi-Bellman equations. Math. Comp., Vol. 76 (2007), No. 260, 1861-1893. MR2336272 (2008i:65161)

[2] L.A. Caffarelli and M. Milman, "Monge Ampère Equation: Applications to Geometry and Optimization", Providence, RI: Amer. Math. Soc., 1999. MR1660738 (99f:00018)

[3] Hongjie Dong and N.V. Krylov, The rate of convergence of finite-difference approximations for parabolic Bellman equations with Lipschitz coefficients in cylindrical domains, Applied Math. and Optimization, Vol. 56 (2007), No. 1, 37-66. MR2334605 (2008e:65240)

[4] W.L. Fleming and H.M. Soner, "Controlled Markov processes and viscosity solutions", second edition, Stochastic Modelling and Applied Probability, 25. Springer, New York, 2006. MR2179357(2006e:93002)

[5] N.V. Krylov, On control of the solution of a stochastic integral equation with degeneration, Izvestiya Akademii Nauk SSSR, seriya matematicheskaya, Vol. 36 (1972), No. 1, 248261 in Russian; English translation: Math. USSR Izvestija, Vol. 6 (1972), No. 1, 249-262. MR0307342(46:6462)

[6] N.V. Krylov, "Nonlinear elliptic and parabolic equations of second order", Nauka, Moscow, 1985 in Russian; English translation Reidel, Dordrecht, 1987. MR815513 (87h:35002)

[7] N.V. Krylov, Unconditional solvability of the Bellman equation with constant coefficients in convex domains, Math. Sbornik, Vol. 135 (1988), No. 3, 297-311 in Russian; English translation: Math.USSR-Sb., Vol. 63 (1989), No. 2, 289-303. MR.937642 (89h:35093)

[8] N.V. Krylov, On the rate of convergence of finite-difference approximations for Bellman's equations, Algebra i Analiz, St. Petersburg Math. J., Vol. 9 (1997), No. 3, 245-256. MR $1466804(98 \mathrm{~h}: 49033)$

[9] N.V. Krylov, Approximating value functions for controlled degenerate diffusion processes by using piece-wise constant policies, Electronic Journal of Probability, Vol.4 (1999), paper no. 2, 1-19, http://www.math.washington.edu/ ejpecp/EjpVol4/paper2.abs.html. MR.1668597 (2000b:49056)

[10] N.V. Krylov, On the rate of convergence of finite-difference approximations for Bellman's equations with variable coefficients, Probab. Theory Relat. Fields, Vol. 117 (2000), No. 1, 1-16. MR1759507 (2001j:65134) 
[11] N.V. Krylov, The rate of convergence of finite-difference approximations for Bellman equations with Lipschitz coefficients, Applied Math. and Optimization, Vol. 52 (2005), No. 3, 365-399. MR2174020 (2006k:65219)

[12] N.V. Krylov, A priori estimates of smoothness of solutions to difference Bellman equations with linear and quasi-linear operators, Math. Comp., Vol. 76 (2007), 669-698. MR2291833 (2008e:65250)

[13] N.V. Krylov, On factorizations of smooth nonnegative matrix-values functions and on smooth functions with values in polyhedra, Appl. Math. Optim., Vol. 58 (2008), No. 3, 373-392. MR2456852 (2009j:15048)

[14] N.V. Krylov, On a representation of fully nonlinear elliptic operators in terms of pure second order derivatives and its applications, Problems of Mathematical Analysis in Russian; English translation: Journal of Mathematical Sciences, New York, Vol. 177 (2011), No. 1, 1-26.

[15] N.V. Krylov, Interior estimates for the first-order differences for finite-difference approximations for elliptic Bellman's equations, Applied Math. Optim., Vol. 65 (2012), No. 3, 349-370. MR.2902696

[16] N.V. Krylov, On the rate of convergence of difference approximations for uniformly nondegenerate elliptic Bellman's equations, submitted to Math. Comp.

[17] H.J. Kushner and P. Dupuis, "Numerical methods for stochastic control problems in continuous time", second edition, Applications of Mathematics (New York), 24. Stochastic Modelling and Applied Probability, Springer-Verlag, New York, 2001. MR1800098 (2001g:93002)

127 Vincent Hall, University of Minnesota, Minneapolis, Minnesota 55455

E-mail address: krylov@math.umn.edu 